\title{
Autocompensating Measurement-Device- Independent Quantum Cryptography in Space Division Multiplexing Optical Fibers
}

Jesús Liñares ( $\nabla$ suso.linares.beiras@usc.es )

Universidade de Santiago de Compostela https://orcid.org/0000-0002-8296-7332

Gabriel M. Carral

University of Santiago de Compostela: Universidade de Santiago de Compostela

\section{Xesús Prieto-Blanco}

University of Santiago de Compostela: Universidade de Santiago de Compostela

Daniel Balado

University of Santiago de Compostela: Universidade de Santiago de Compostela

\section{Research}

Keywords: quantum cryptography, measurement-device-independent, space division multiplexing, autocompensation, integrated quantum optics

Posted Date: January 9th, 2021

DOl: https://doi.org/10.21203/rs.3.rs-141385/v1

License: (9) (1) This work is licensed under a Creative Commons Attribution 4.0 International License. Read Full License 


\title{
Autocompensating Measurement-Device-Independent quantum cryptography in space division multiplexing optical fibers
}

\author{
J. Liñares ${ }^{*}$, G M. Carral, X. Prieto-Blanco and D. Balado
}

\begin{abstract}
Single photon or biphoton states propagating in optical fibers or in free space are affected by random perturbations or imperfections along optical fibers or free space that disturb the information encoded in such states and accordingly quantum key distribution is prevented. We propose three different systems for autocompensating such random perturbations and imperfections when a measurement-device-independent protocol is used. These systems correspond to different optical fibers intended for space division multiplexing and supporting collinear modes, polarization modes or codirectional modes such as few-mode optical fibers and multicore optical fibers. Accordingly, we propose different Bell-states measurement devices. Finally, these types of optical fibers allow the use of several transmission channels what compensates the reduction of the bit rate due to losses.
\end{abstract}

Keywords: quantum cryptography; measurement-device-independent; space division multiplexing; autocompensation; integrated quantum optics

\section{Introduction}

Over the last few years, space division multiplexing has been proposed to further increase the data bandwidth in optical communications; thus, high interest has arisen about new optical fibers such as few-mode fibers (FMFs) [1] and multicore fibers (MCFs) [2]. Consequently, the interest in the implementation of quantum cryptography in these new optical fibers has remarkably increased in the last few years. Quantum cryptography states that the laws of quantum mechanics, if correct, guarantee unconditional security of communications under quantum key distribution protocols $[3,4]$. At least, this is what theory teaches us. When it comes to real-life implementations, however, a number of problems arise that put such assertion in jeopardy. This is due to the fact that devices used in Quantum Key Distribution (QKD) protocols are imperfect in reality. Such imperfections open a backdoor for an eavesdropper (Eve) to obtain information that ideally

\footnotetext{
${ }^{*}$ Correspondence: suso.linares.beiras@usc.es

Quantum Materials and Photonics Research Group, Optics Area, Applied Physics Department, Faculty of Physics / Faculty of Optics and Optometry, University of Santiago de Compostela, Campus Vida s/n, E-15782 Santiago de Compostela, Galicia, Spain

Full list of author information is available at the end of the article
}

would be perfectly concealed. One of the most important problems corresponds to the detector side channel attacks. They are related to the fact that the detector apparatuses Alice and/or Bob use may be flawed, and that might give Eve an opportunity to extract secret information about the key without Alice and Bob knowing $[5,6,7]$. Fortunately for the security of communications, a quite good solution has been developed, that is, the Measurement-Device-Independent quantum key distribution (MDI-QKD) [8]. This idea consists in making use of entanglement in such a way that the whole measurement process is treated like a black box. Traditionally, in QKD, the measurement is done by the partner sitting at the end of the line (normally Bob). In this case, a third party, called Charlie, makes the measurement. He performs a Bell measurement on the two-qubit state Alice and Bob send him. The key is that Charlie may be untrusted. He can be an eavesdropper himself. Charlie is only asked to report whether he obtained a successful or an unsuccessful result from his measurement, and he can of course lie. May the detectors he uses be flawed and/or may he be Eve in disguise: it does not matter. The detection process is a black box, what happens inside has 
no relevance, only what Charlie reports in relation to what quantum state Alice and Bob sent. To this last part, usual defence methods need to be applied (i.e. decoy states [9], etc). Thus, Alice and Bob can establish a secret key without the need to take into account detector side channels attacks. The main downside of this solution is that the key rate is small [10] compared to standard protocols. Also, sources need to be trusted and state preparation has to be almost perfect. There also other practical problems to overcome [11]. But, in all, among the proposals that inherently protect against side-channel attacks, MDI-QKD is the one of greater feasibility $[4,8]$.

Another class of practical problems in quantum (and classical) communications are the perturbations that photons suffer when they propagate along optical fibers. Certain perturbations and also imperfections in practical optical fibers randomly scramble the information encoded in photons, impairing QKD. When the link is very short this posses no real problem, but in real-life quantum communications links are required to be about a few hundreds of kilometres, and the aim is precisely to increase this limit. An example of such pertubations is the undesired birefringence most real-life fibers have, unless they are polarizationmaintaining fibers [12], which have a high but tailored built-in birefringence, so as to preserve two particular linear polarization states of the travelling photons although the relative phase remains random. Information may be encoded in the photons polarization, and undesired birefringence alters the state of polarization unpredictably. In other words, such birefringence cannot be completely characterized, causing a random perturbation. Then, if polarization changes randomly when the photons are on the fly, no key can be established. On the other hand, in optical fibers intended for space division multiplexing, spatial perturbations, due to mechanical causes, thermal causes and so on, can also give rise to undesired and unpredictable couplings between spatial modes (modal crosstalk) with the same effect of precluding QKD. This last case is acute when one deals with many spatial modes and multicore optical fibers (MCFs) and even few-mode fibers (FMFs). Moreover, these spatial perturbations can also change with time although slowly respect to transmission speed. Likewise, imperfections in optical fiber can give rise to mode coupling. Solutions to such problems have been proposed in QKD protocols by the so-called autocompensating cryptography, thus, autocompensating QKD methods have been proposed for single photon states excited in polarization and/or spatial modes [13, 14]. Autocompensating cryptography consists in taking the travelling light and make it go through some determinate optical elements that alter its state in such a way that the perturbations become harmless. The price to pay is that light has to travel back-and-forth between the two ends of the line, that is, it has to travel at least two times the same distance. This enhances distance-related drawbacks like fiber losses. Note that autocompensation does not eliminate or make any attempt to eliminate such perturbations, but their effects, restoring the photonic quantum state that was originally launched. At this point we must stress that in optical fibers for space division multiplexing several channels could be used, thus reducing the effects associated to fiber losses. Another solution exists, consisting in continuously monitoring the properties of the communication links like optical fibers, correcting in real time the undesired modifications the propagating state can undergo as for example relative phases, although coupling effects would be much more difficult to correct [15].

In this work, we propose an Autocompensating MDI-QKD (A-MDI-QKD) protocol, which is based on biphoton states. We will do it in three ways, each one corresponding to a different photonic encoding: collinear modes, polarization modes and codirectional modes. By collinear modes we refer to modes propagating along the same direction, travelling in the same core of a fiber, such as Hermite-Gauss (HG) modes. Few mode optical fibers are the transmission lines for collinear modes, although optical communication in free space can make use of this kind of spatial modes. We will distinguish between polarization-maintaining few mode fibers (PM-FMFs) [12], where two particular linear polarizations are maintained long distances, and space-maintaining few-mode fibers (SM-FMFs), where spatial modes are maintained decoupled long distances [16]. By codirectional modes, we refer to modes travelling along different fibers or different cores of the same fiber. This kind of modes are directly compatible with integrated optics by using photonic lanterns or proper optical connectors. As commented, nowadays, research in MCFs is an active field, since MCFs can be used to increase to a large extent the communication capacities of fiber links. Moreover, this makes the adaptability to current (and future) systems a lot easier, with the possibility of using the available infrastructure to set up A-MDI-QKD protocols following a plug-andplay philosophy [17]. On the other hand, scalability, compactness, robustness.., are well-known attributes of optical integrated technologies [18]. Importantly, integrated devices, together with MCFs [19, 20], open the possibility, in a very clear way, to extend the protocol to $N$ dimensions, which increases security. At this point, we must also stress that the use of mode converters based on photonic lanterns [21] or multi-plane interfaces [22] between FMFs and channel waveguide 
modes also allow to use integrated optical components because collinear modes can be converted into codirectional modes, although in this work, for the sake of completeness, bulk components will also be used. The same interface procedure can be applied to polarization modes. We will use four modes where two photons are excited, that is, two-qubits. Beisdes, as the algebraic mechanism to obtain autocompensation is different for each encoding, then we will deal with each case separately. Therefore, the plan of this paper is as follows. We start by proposing a full A-MDIQKD optical system for collinear modes coming from PM-FMFs, which can provide several spatial channels for QKD because polarization is maintained a long distance. Next, a A-MDI-QKD optical system for polarization modes of SM-FMFs is presented. Moreover, as in the spatial case it can also provide several polarization QKD channels because spatial modes are maintained decoupled for a long distance. Finally, we propose A-MDI-QKD optical system for codirectional modes coming from MCFs by using integrated optics to measure the Bell states. The main conclusions are summarized at the end.

\section{A-MDI-QKD system with collinear modes}

As commented, for this case, we encode information in two collinear spatial modes of two PM-FMFs linking Alice-Charlie and Bob-Charlie as shown in Fig. 1. This kind of fibers have a high birrefringence in such a way that polarization modes are well separated, however spatial modes can undergo coupling. For instance in this kind of fibers, there will be mode coupling between $\mathrm{LP}_{11 H}^{(e)}$ and $\mathrm{LP}_{11 H}^{(o)}$ modes, and $\mathrm{LP}_{11 V}^{(e)}$ and $\mathrm{LP}_{11 V}^{(o)}$ modes [12], with (e) indicating even mode $(\cos \varphi)$ and (o) odd mode $(\sin \varphi)$. However, polarization mode coupling is negligible. Obviously, each polarization can be considered as a channel potentially useful for QKD, therefore, we could use several independent channels to perform QKD, which would increase the bit rate and therefore would reduce the loss effects and larger distances would be achieved. For example, let us consider without loss of generality that the quantum states are excited in the particular modes $\operatorname{LP}_{11 H}^{(e)} \equiv X$ and $\mathrm{LP}_{11 H}^{(o)} \equiv Y$, that is, in horizontal $(\mathrm{X})$ and vertical $(\mathrm{Y})$ Hermite-Gaussian (HG) modes polarized, for instance, along the horizontal direction. Single photon states are denoted as $\left|1_{X}\right\rangle$ and $\left|1_{Y}\right\rangle$. The vertical polarization direction could be used as a second channel. More channels could be considered if other modes are used as, for example, $\mathrm{LP}_{21 H}^{(e)}$ and $\mathrm{LP}_{21 H}^{(o)}$ modes, and $\mathrm{LP}_{21 V}^{(e)}$ and $\mathrm{LP}_{21 V}^{(o)}$ modes [12].

The A-MDI-QKD system is shown in Figure 1. As for autocompensation light needs to go back-and-forth, then light must start its way at Charlie. Lighjt can come from a SPDC or two independent lasers emitting WCPs (weak coherent pulses) and ensuring photon indistiguishability, Charlie prepares the following state

$$
\left(\frac{\left|1_{X}\right\rangle+\left|1_{Y}\right\rangle}{\sqrt{2}}\right)_{a} \otimes\left(\frac{\left|1_{X}\right\rangle+\left|1_{Y}\right\rangle}{\sqrt{2}}\right)_{b}
$$

and sends each part of it to Alice and Bob. Indexes $a$ and $b$ stand for Alice's and Bob's paths, respectively. If WCPs are used then decoy states are also required in order to prevent photon number splitting (PNS) attacks. We group whatever source Charlie uses to generate those states under the name Initial States Generator (ISG). Next, light reaches Charlie's optical circulators (OC), which direct the light towards Alice's and Bob's sites. After the circulators optical fiber delay (OFD) devices are placed to delay states excited in mode $\mathrm{Y}$ a time $\tau$ with respect to states excited in mode $X$, meaning that we will have $\mid 1_{X}$ and $\left|1_{Y \tau}\right\rangle$. The OFDs (see Fig. 2) consist on a Mach-Zehnder in-

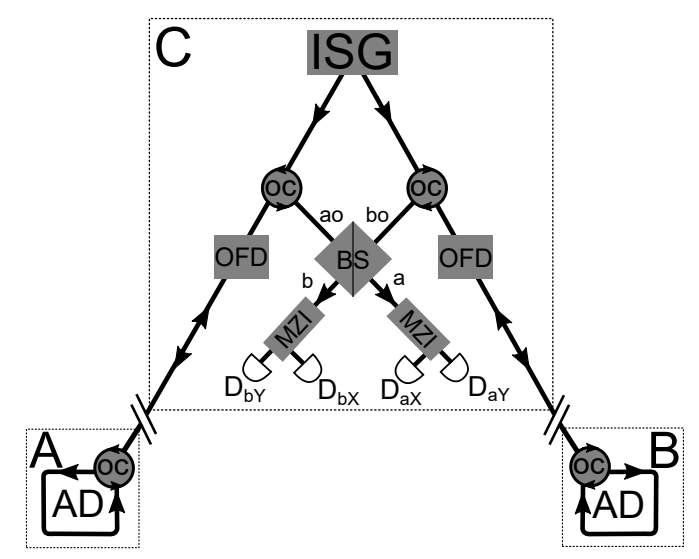

Figure 1 A-MDI-QKD system for collinear modes. Light comes from Charlie along PM-FMF optical fibers. States are delayed by OFDs. They arrive at Alice's and Bob's

laboratories, where they travel along local loops equipped with autocompensating devices (AD) and phase modulators. Local loop structure is symbolized by a loop with $A D$ inside. Light returns to Charlie, and optical circulators (OC) direct the photons towards a Bell-state measurement apparatus composed by a BS and two MZIs working as mode sorters, together with the corresponding detectors.

terferometer (MZI) with a Dove prism (DP) in one of its arms. They work in the following way. When the quantum state reaches the first beam splitter (BS), one part of it goes into the horizontal $(h)$ path and another on the vertical $(v)$ path. Then, the Dove prism in the vertical path introduces the following transformation 
on the modes

$$
D_{\vartheta}=\left(\begin{array}{cc}
\cos 2 \vartheta & \sin 2 \vartheta \\
\sin 2 \vartheta & -\cos 2 \vartheta
\end{array}\right)
$$

where $\vartheta$ is the angle the normal of the prism is rotated. This means that if we have states $\left|1_{X}\right\rangle$ and $\left|1_{Y}\right\rangle$ as input, the output will be a $\left|1_{X^{\prime}}\right\rangle$ mode given by the linear combination $\left|1_{X^{\prime}}\right\rangle=\cos 2 \vartheta\left|1_{X}\right\rangle+\sin 2 \vartheta\left|1_{Y}\right\rangle$, and analogously for $\left|1_{Y}\right\rangle$. Setting $\vartheta=0$ we obtain the transformations $\left|1_{X^{\prime}}\right\rangle=\left|1_{X}\right\rangle$ and $\left|1_{Y^{\prime}}\right\rangle=-\left|1_{Y}\right\rangle$. So, for an input $\frac{1}{\sqrt{2}}\left(\left|1_{h X}\right\rangle+\left|1_{h Y}\right\rangle\right)$ at the horizontal input of the first BS, the emergent state after the second BS is $\frac{1}{\sqrt{2}}\left(\left|1_{v X}\right\rangle-\left|1_{h Y}\right\rangle\right)$. This means that the $X$ mode exits the vertical port of the second BS and is directed towards Alice (and Bob), while the $Y$ mode exits the horizontal port, and is directed towards a external optical path that feeds the vertical port of the first BS. The result is that the $Y$ mode gets delayed with respect to the $X$ mode, by a quantity we call $\tau$. This delay will allow Alice and Bob to introduce relative phases between $X$ and $Y$ modes. Thus, the state after the OFDs is

$$
\left|L_{e}\right\rangle=\left(\frac{\left|1_{X}\right\rangle+\left|1_{Y \tau}\right\rangle}{\sqrt{2}}\right)_{a} \otimes\left(\frac{\left|1_{X}\right\rangle+\left|1_{Y \tau}\right\rangle}{\sqrt{2}}\right)_{b}
$$

Now, photons enter (and then exit) optical fiber cir-

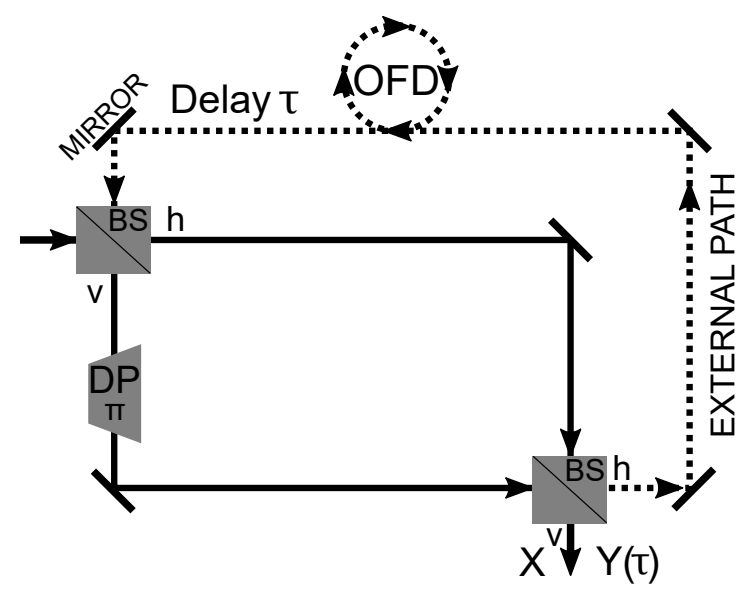

Figure 2 Scheme of a MZI operating with HG modes. A Mach-Zehnder Interferometer together with a Dove prism in one of its arms operates as a HG mode sorter. At the output of the last $\mathrm{BS}$, the $\mathrm{X}$ mode emerges on the $v$ path and the $Y$ mode on the $h$ path. This modes can be either redirected to detectors, as in Figure 1, to project onto Bell states, or the $Y$ mode can be redirected into a external path (dashed line), introducing a delay $\tau$ between modes, that is, $\left|1_{X}\right\rangle$ and $\left|1_{Y \tau}\right\rangle$. Thus, such MZls can be used as 'polarizing' beam splitters (thinking of $X$ and $Y$ as polarizations) for Bell-state detection as well as OFD devices. cuits at Alice and Bob's sites trough optical circulators. Note that Alice and Bob's laboratories need to be well shielded from eavesdroppers. No information should leak out. In these circuits, autocompensating devices are located, together with phase modulators by which Alice and Bob encode their bits. Electro-optical phase shifters implement this task. Alice and Bob introduce a relative phase $e^{i \theta}$ between $\left|1_{X}\right\rangle$ and $\left|1_{Y}\right\rangle$, randomly chosen from the set $\theta=\{-\pi / 2,0, \pi / 2, \pi\}$, in order to encode information in one of the two following mutually unbiased bases (MUBs), that is, diagonal and circular bases,

$$
\begin{gathered}
\mathcal{B}_{D}:\left\{\left|1_{D}\right\rangle=\frac{\left|1_{X}\right\rangle+\left|1_{Y}\right\rangle}{\sqrt{2}},\left|1_{A}\right\rangle=\frac{\left|1_{X}\right\rangle-\left|1_{Y}\right\rangle}{\sqrt{2}}\right\}, \\
\mathcal{B}_{C}:\left\{\left|1_{L}\right\rangle=\frac{\left|1_{X}\right\rangle+i\left|1_{Y}\right\rangle}{\sqrt{2}},\left|1_{R}\right\rangle=\frac{\left|1_{X}\right\rangle-i\left|1_{Y}\right\rangle}{\sqrt{2}}\right\} .
\end{gathered}
$$

It is worth to writing the transformation between absorption operators $\hat{a}$ in both bases, that is

$$
\hat{a}_{\left(\begin{array}{c}
D \\
A
\end{array}\right)}=\frac{1}{\sqrt{2}}\left(\hat{a}_{X} \pm \hat{a}_{Y}\right) ; \quad \hat{a}_{\left(\begin{array}{l}
L \\
R
\end{array}\right)}=\frac{1}{\sqrt{2}}\left(\hat{a}_{X} \mp i \hat{a}_{Y}\right)
$$

Note again that autocompensation requires light to follow a closed path. As in MDI-QKD the photons end at Charlie's site, if we add autocompensation, light needs to start its way at Charlie's site too. Charlie provides the input light and Alice and Bob modulate its phase in order to generate quantum states on which encode their bits. It is clear why we can not use the usual basis $\left\{\left|1_{X}\right\rangle,\left|1_{Y}\right\rangle\right\}$ (socalled the $\mathrm{Z}$ or rectilinear basis and used normally in MDI-QKD protocol [8]) because it can not be converted in any other basis by phase modulation. Obviously, rotations can be used to change basis, but, in that case, the autocompensating transformation we will describe afterwards would become useless, hence autocompensation would not work. We will ignore the perturbations for a while to explain the measurement process, then we will deal with the autocompensation method.

\section{Bell-state measurement device}

After exiting the fiber circuits, light goes back to Charlie. At Charlie's circulators light is directed towards a Bell-state measurement device. This device is composed by a beam splitter (BS) plus two MZI identical to the MZIs present above in Fig. 2 (except that they lack the external optical path for OFD). The BS acts the usual way, entangling photons and producing Bell states, which will be postselected afterwards. As analyzed, the MZIs act as a polarizing beam splitter would do in the polarization case, that is, they sort $X$ and $Y$ modes, each one in one different port, 
as we have seen. Then, photons are directed towards detectors $D_{a X}, D_{a Y}, D_{b X}, D_{b Y}$ and Bell states will be measured. In detail, the working principle of this Bellstate measurement apparatus is the following. Let's call the input ports of the BS after the OCs in Figure 1 as $a o$ and $b o$, and the output ports as $a$ and $b$. Now, assume that such BS is of the type that induces the following transformation between the input and output modes, with corresponding photon emission operators $\hat{a}_{a o}^{\dagger}, \hat{a}_{b o}^{\dagger}, \hat{a}_{a}^{\dagger}, \hat{a}_{b}^{\dagger}$ :

$$
\hat{a}_{a o}^{\dagger}=\frac{\hat{a}_{a}^{\dagger}+i \hat{a}_{b}^{\dagger}}{\sqrt{2}} ; \quad \hat{a}_{b o}^{\dagger}=\frac{i \hat{a}_{a}^{\dagger}+\hat{a}_{b}^{\dagger}}{\sqrt{2}} .
$$

Now, when Alice and Bob choose the same basis (else the data is discarded), there are two classes of input states. The first one is when Alice and Bob prepare the same state and the second one is when they prepare different states. Consider the first case of a diagonal basis. Let us consider, for instance, that the state that reaches the $\mathrm{BS}$ is

$$
\left|L_{n f}\right\rangle=\left|1_{a o D}\right\rangle\left|1_{b o D}\right\rangle
$$

This state corresponds to the case so-called not bitflip case. This state can be rewritten, by taking into account the transformation given by Eq. (7), as follows

$$
\left|L_{n f}\right\rangle=\frac{i}{2}\left(\hat{a}_{a D}^{\dagger 2}+\hat{a}_{b D}^{\dagger 2}\right)|00\rangle
$$

Next, by using the measurement modes $X$ and $Y$ we obtain

$$
\left|L_{n f}\right\rangle=\frac{i}{4}\left\{\left(\hat{a}_{a X}^{\dagger}+\hat{a}_{a Y}^{\dagger}\right)^{2}+\left(\hat{a}_{b X}^{\dagger}+\hat{a}_{b Y}^{\dagger}\right)^{2}\right\}|00\rangle
$$

Note that by one hand we obtain the following state

$$
\left|L_{n c}\right\rangle=\frac{\sqrt{2}}{4}\left(\left|2_{a X}\right\rangle+\left|2_{a Y}\right\rangle+\left|2_{b X}\right\rangle+\left|2_{b Y}\right\rangle\right)
$$

that is, we detect the two photons in only one detector, that is, there are no coincidences with a probability equal to $1 / 2$. On the other hand, we also obtain with a probability equal to $1 / 2$, the following Bell state $\Phi^{+}$ (with respect to the detectors $a$ and $b$ ),

$$
\left|L_{c}\right\rangle=\frac{\sqrt{2}}{2}\left|\Phi^{+}\right\rangle=\frac{\sqrt{2}}{2} \frac{\left(\left|1_{a X} 1_{a Y}\right\rangle+\left|1_{b X} 1_{b Y}\right\rangle\right)}{\sqrt{2}}
$$

meaning that there are coincidences in the same output, $a$ or $b$, that is, there will be a a simultaneous click between the same pair of detectors, either $D_{a X}$ together with $D_{a Y}$ or $D_{b X}$ together with $D_{b Y}$. However, if the input state is, for instance,

$$
\left|L_{f}\right\rangle=\left|1_{a o D}\right\rangle\left|1_{b o A}\right\rangle
$$

which corresponds to a bit-flip, then by taking into account the transformation given by Eq. (7), the the state can be rewritten as

$$
|L\rangle=\frac{i}{2}\left(\hat{a}_{a D}^{\dagger}+i \hat{a}_{b D}^{\dagger}\right)\left(i \hat{a}_{a A}^{\dagger}+\hat{a}_{b A}^{\dagger}\right)|00\rangle
$$

By following the same procedure than in the above case, we obtain both states corresponding to non coincidences and the following Bell state, with a probability equal to $1 / 2$,

$$
\left|L_{c}\right\rangle=\frac{\sqrt{2}}{2}\left|\Psi^{-}\right\rangle=\frac{\sqrt{2}}{2} \frac{\left(\left|1_{a X} 1_{b Y}\right\rangle-\left|1_{b X} 1_{a Y}\right\rangle\right)}{\sqrt{2}}
$$

that is, coincident clicks between $D_{a X}$ and $D_{b Y}$ or $D_{a Y}$ and $D_{b X}$, are obtained, which is precisely the $\left|\Psi^{-}\right\rangle$ Bell state. Now, each photon emerges in a different port. We must stress that the non coincidences can be used to calibrate the system, that is, we must detect the same amount of coincidences and non coincidences to make sure that the system is aligned and adjusted and therefore the protocol can be implemented. However, we still need to eliminate the effect of possible perturbations by an autocompensating method.

\section{Autocompensation method}

As commented, we encode information in two collinear spatial modes propagating in a PM-FMF along $z$ direction, in particular, $\mathrm{LP}_{11 H}^{(e)} \equiv X$ and $\mathrm{LP}_{11 H}^{(o)} \equiv Y$. We will describe in detail the autocompensation mechanism that enables to get rid of perturbations that arise when such modes propagate along optical fibers. The aim of autocompensation is that the photonic state emerges restored after it has travelled the path, back and forth, between the starting point and the endpoint of the fiber link. Intrinsic imperfections of dielectric permitivitty or perturbations $P(x, y)$ produced by mechanical and/or thermal causes give rise to modal coupling between optical modes. The two-mode coupling coefficient is given by

$$
\kappa_{s}=\int e_{1}(x, y) P(x, y) e_{2}(x, y) d x d y
$$

where $e_{1}(x, y)$ and $e_{2}(x, y)$ are the mode amplitudes. $P(x, y)$ is a random perturbation, which can regarded 
as $z$-invariant in a short propagation distance $z$. Therefore the total perturbation is a sequence of $q$ perturbations with different coupling coefficients. Each perturbation $k=1, \ldots q$ can be described by an asynchronous modal coupling, that is, the formal solution for the absorption operators is given by

$$
\left(\begin{array}{l}
\hat{a}_{k 1} \\
\hat{a}_{k 2}
\end{array}\right)=S_{k}\left(\begin{array}{l}
\hat{a}_{k 01} \\
\hat{a}_{k 02}
\end{array}\right)=\left(\begin{array}{cc}
A_{k} & i B_{k} \\
i B_{k} & A_{k}^{*}
\end{array}\right)\left(\begin{array}{l}
\hat{a}_{k 01} \\
\hat{a}_{k 02}
\end{array}\right)
$$

Now, we can model the perturbations along the fiber as a discrete number $q$ of perturbations, acting on the absorption operators. Moreover, since we are dealing with single-photon states, it can be shown that the solutions to the Heisenberg equations for spatial propagation apply directly to photonic states [23]. Thus, if $|\psi\rangle$ is a photonic state, then $|\psi(z)\rangle=S|\psi(0)\rangle$, where $S$ is the total matrix $S=S_{q}, S_{q-1} \ldots S_{2} S_{1}$.

On the other hand, the coupling coefficients remain invariant under backpropagation, that is, by defining a new reference system given, for instance, by $(-X) Y(-Z)$, the original one being given by $X Y Z$, it is easy to check that $\kappa_{s}$ does not change under backpropagation, therefore, the matrices $S_{k}$ do not change under backpropagation. We write down explicitly the transformation the photonic states would experience when going back-and-forth along the optical fiber. When going from Charlie to Alice (and Bob), we have $T_{\rightarrow}=S_{q} \ldots S_{1}$, and, when going back from Alice (Bob) to Charlie, we have again $T_{\leftarrow}=S_{1} \ldots S_{q}$. Between these two operations, we have to put the right transformation, implemented by the adequate autocompensating devices, so that we restore the original photonic state. The full transformation the light experiences on a roundtrip is

$$
T_{\leftarrow} M T_{\rightarrow}=S_{1} \ldots S_{q} M S_{q} \ldots S_{1}
$$

where $M$ represents the operation of the autocompensating devices. It is easy to check that $M=i \sigma_{y}$, with $\sigma_{y}$ the second Pauli matrix, restore the state that is

$$
S_{k} M S_{k}=\left(\begin{array}{cc}
A_{k} & i B_{k} \\
i B_{k} & A_{k}^{*}
\end{array}\right)\left(\begin{array}{cc}
0 & 1 \\
-1 & 0
\end{array}\right)\left(\begin{array}{cc}
A_{k} & i B_{k} \\
i B_{k} & A_{k}^{*}
\end{array}\right)
$$

then $S_{k} M S_{k}=M$. This means that the state of the emergent light, back at Charlie's circulators, has been properly restored, albeit a deterministic swapping transformation $M=i \sigma_{y}$, which we can account for.

Next, we must answer now the question of how to implement the operation $M=i \sigma_{y}$ with HG modes. This can be done using two cylindrical lens converters (CLCs) [24] and a Dove prism with $\vartheta=\pi / 4$, plus a phase shifter (PS) introducing a global phase $\phi=-\pi / 2$, as shown in Figure 3 . The Dove prism is sandwiched between the CLCs. The CLCs rotate the $X$ and $Y$ modes by phases $\pm \pi / 2$, respectively. They convert Hermite-Gauss modes into LaguerreGauss ones. In matrix form, this can be written as

$$
C L C_{ \pm \pi / 2}=\left(\begin{array}{cc}
1 & 0 \\
0 & \pm i
\end{array}\right)
$$

Now, the Dove prism is rotated $\pi / 4$, therefore, from (2), it implements the operation $D_{\pi / 4}=\sigma_{x}$. Therefore, we obtain the desired transformation,

$$
M=e^{-i \pi / 2} C L C_{-\pi / 2} D_{\pi / 4} C L C_{\pi / 2}=\left(\begin{array}{cc}
0 & 1 \\
-1 & 0
\end{array}\right)
$$

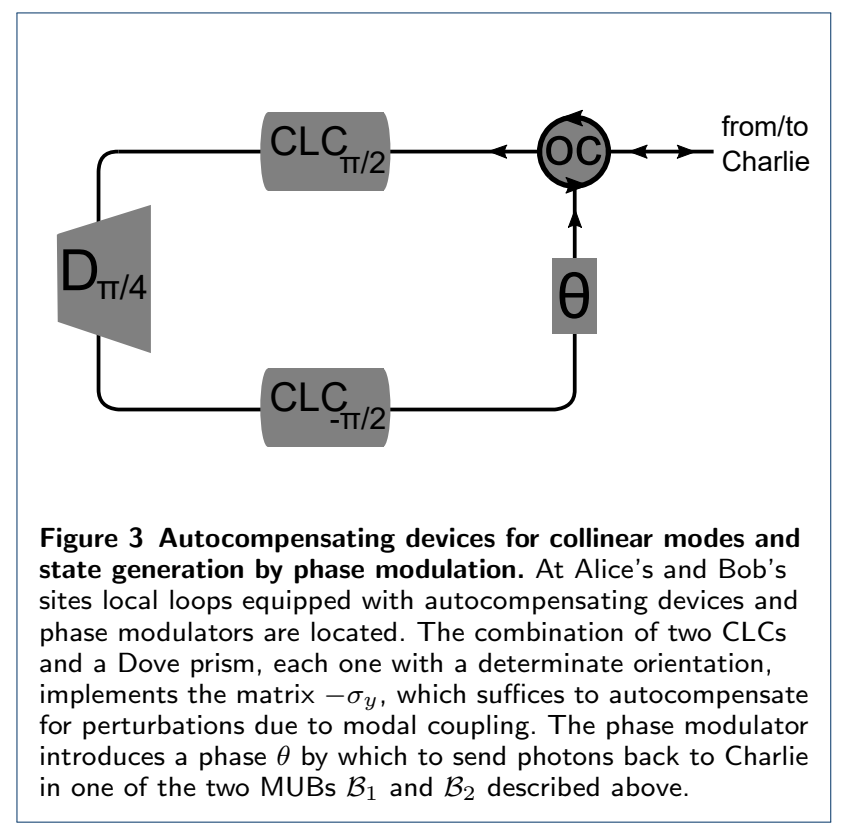

We left some more nuances along the road that deserve however more attention. First of all, when we wrote (18), it may seem that we forgot about the phase gate Alice and Bob apply in order to obtain the different quantum states in which encode their bits. However, we must recall that at Alice and Bob systems the states are excited in retarded modes as given by Eq. (3). In other words, autocompensation is for the retarded states $\left|1_{X}\right\rangle$ and $\left|1_{Y \tau}\right\rangle$ in an individualized fashion. The transformation $U(\theta)=\operatorname{diag}\left(1, e^{i \theta}\right)$ implementing the phase modulation acts on the above states, in particular on $\left|1_{Y \tau}\right\rangle$. Another subtlety is that the fiber circuits, where the autocompensation devices and phase modulators are located, have a very small length. Note that this path is never autocompensated 
but is however so small that is contribution to a perturbation of the photonic states is negligible. They are local fiber loops. We remark that this will be common to all three encodings described in this paper. Moreover, we must note that the delay introduced in OFD when the photons come back cancels the delay introduced at the beginning. This is because the Dove- $\pi / 4$ prism formally swaps modes $X$ and $Y$ but does not swap their identity. This implies that, if when the photons first went through the OFD, the $X$ mode went ahead of $Y$, then after the Dove- $\pi / 4$ prism, it is the $Y$ mode who goes ahead. Then, again at the OFD device, the $Y$ gets retarded and waits for the $X$ mode so that they reach Charlie's Bell state apparatus exactly at the same time. This can be calibrated by using the HOM states that are produced at the BS.

Finally, note that we are saying that modes coming from Alice's and modes coming from Bob's reach the Bell-state projector at the same time, but separately, in the sense that the $\mathrm{X}$ mode of Alice (Bob) and the $\mathrm{Y}$ mode of Alice (Bob) become synchronous again once they have travelled the OFD twice, but there is still needed that Alice's modes and Bob's modes reach the measurement apparatus at the same time. For that case, the ber paths need to be adjusted to avoid mismatch [11]. Alternatively, if WCPs are used, the pulses generated at the ISG could be delayed such that they return simultaneously. This calibration task is simpler than in a standard configuration as Charlie can perform it without the involvement of Alice or Bob.

\section{A-MDI-QKD system with polarization modes}

For the sake of completeness, we describe the polarization encoding scheme. Polarization is perhaps the best known and most used of all possible photonic encodings, and A-MDI schemes involving it have already been proposed [25]. Still, we describe it here for two reasons apart from the mentioned completeness. The first one is because we do it using a different autocompensating device (a closed cycle with a HWP) to the one used in reference [25] where Farday mirrors are used. The second, and most important, is because in SM-FMF for space division multiplexing, as for example an elliptical FMFs, spatial modes are separated but polarization modes are close [26]. Therefore in these optical fibers we can use these separated space modes as different channels with two polarization modes to perform QKD. Then, as in the above case, we could use several independent channels to carry out QKD, which would increase the bit rate and thus would reduce the loss effects and larger distances would be achieved in QKD.
The basic scheme of the AD suystem is shown in Fig. 4. A biphoton source or two independent lasers producing WCP is required at Charlie's site, together with decoy states. While the working principle is the same, there will be a number of changes on the optical devices used. Charlie sends the state

$$
\left(\frac{\left|1_{H}\right\rangle+\left|1_{V}\right\rangle}{\sqrt{2}}\right)_{a} \otimes\left(\frac{\left|1_{H}\right\rangle+\left|1_{V}\right\rangle}{\sqrt{2}}\right)_{b},
$$

where again $a$ stands for Alice's path and $b$ for Bob's path. Note that the formal study is identical to the case of spatial modes $X$ and $Y$ but with the formal changes $X \rightarrow H$ and $Y \rightarrow V$. Therefore, the implementation of the MDI protocol is made in the same way and consequently the results found above can be easily used for polarization. However, the autocompensation process will be different. As in the spatial mode

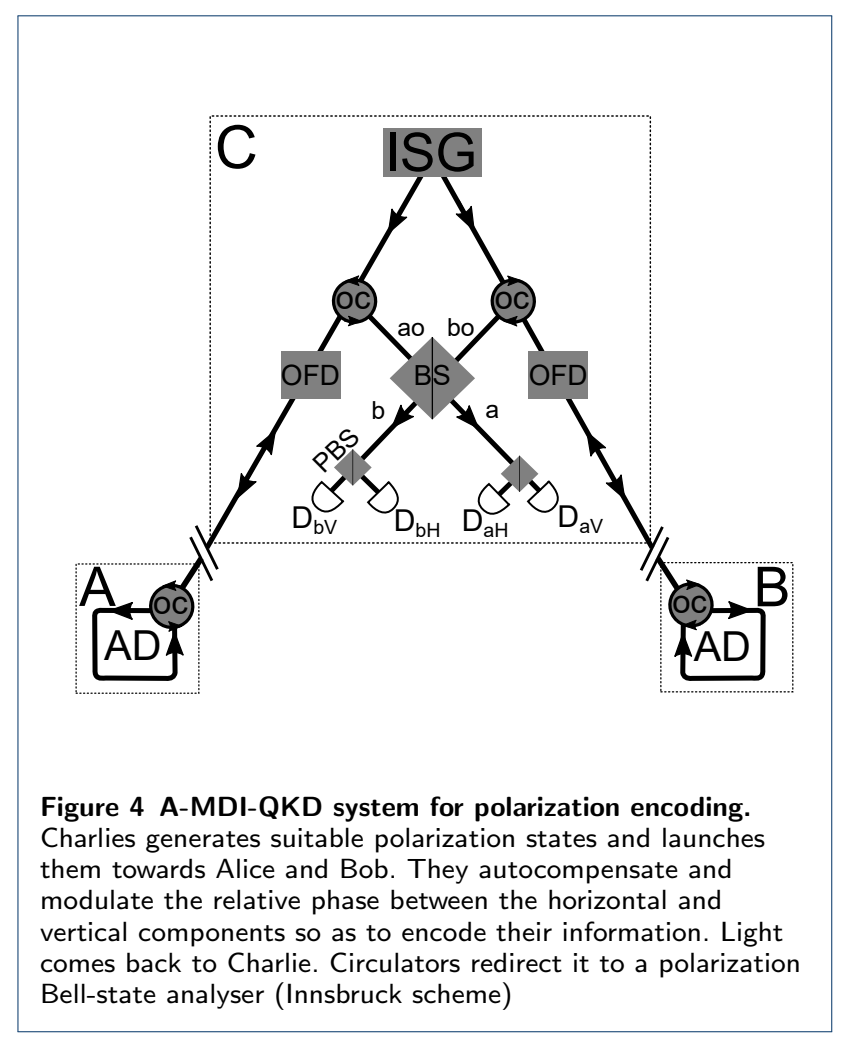

case optical fiber delays are again required. This can be implemented for this case with the aid of a PBS [27], as shown in Fig. 5 a). As we will see, the autocompensating device again implements a swapping operation required to cancel such delay so that states reach Charlie's Bell-state analyzer at the same time. Light goes towards Alice's and Bob's sites, each one equipped with autocompensating devices. Photons enter optical fiber circuits at Alice and Bob's sites trough optical circulators. By means of electro-optical phase shifters, Alice and Bob encode their bits. As before, Alice and Bob 
introduce a relative phase $\theta$ between $\left|1_{H}\right\rangle$ and $\left|1_{V}\right\rangle$, randomly picked from the set $\theta=\{-\pi / 2,0, \pi / 2, \pi\}$, in order to encode information in one of the two MUBs (diagonal or $\sigma_{x}$ and circular or $\sigma_{y}$ ).

After exiting the optical fiber circuits at Alice and Bob's sites, light returns to Charlie. At Charlie's circulators light is directed towards a linear optics system which implements a Bell-state measurement (Innsbruck scheme). At the beam-splitter BS Bell states are produced. Afterwards, two polarizing beam-splitters (PBS) with their corresponding detectors $\mathrm{D}_{a H}, \mathrm{D}_{a V}$ and $\mathrm{D}_{b H}, \mathrm{D}_{b V}$ project into the individual polarizations. In particular, we obtain the following Bell state for the not bit-flip case

$$
\left|\Phi^{+}\right\rangle=\frac{1}{\sqrt{2}}\left(\left|1_{a H} 1_{a V}\right\rangle+\left|1_{b H} 1_{b V}\right\rangle\right),
$$

meaning that there are coincidences in the same output, $a$ or $b$, that is, there will be a a simultaneous click between the same pair of detectors, either $D_{a H}$ together with $D_{a V}$ or $D_{b H}$ together with $D_{b V}$. However, for bit-flip cases we obtain

$$
\left|\Psi^{-}\right\rangle=\frac{1}{\sqrt{2}}\left(\left|1_{a H} 1_{b V}\right\rangle-\left|1_{a H} 1_{b V}\right\rangle\right),
$$

that is, coincident clicks between $D_{a H}$ and $D_{b V}$ or $D_{a H}$ and $D_{b V}$, are obtained.

\section{Autocompensation method}

We assume that the optical fiber has birefringency perturbations acting on polarization states. Moreover, as commented this perturbation can vary in time, but slowly. Such perturbation can be also represented by the matrix $S_{k}$ given by Eq. (17). In fact, it is also an asynchronous coupling but with polarization modes coupled by birefringence. However in this case the coupling coefficient between polarization modes is given by

$$
\kappa_{p}=\int e_{H}(x, y) P(x, y) e_{V}(x, y) d x d y .
$$

Then under backpropagation, that is, by defining a new reference system given, for instance, by $(-X) Y(-Z)$, the original one being given by $X Y Z$, polarization modes must be changed, that is, $H \rightarrow-H$, that is, $e_{H}(x, y) \rightarrow-e_{H}(x, y)$, and accordingly the coupling coefficient $\kappa_{p}$ changes its sign. The main consequence is that matrices $S_{k}^{\prime}$ for backpropagation also change, that is,

$$
S_{k}^{\prime}=\left(\begin{array}{cc}
A_{k} & -i B_{k} \\
-i B_{k} & A_{k}^{*}
\end{array}\right)
$$

These results can easily checked by analysing the matrix of particular wave-plate for backpropagation, for instance, a HWP forming an angle $\gamma$ with respect to the axis $X$, that is,

$$
H W P_{\gamma}=\left(\begin{array}{cc}
-i \cos 2 \gamma & -i \sin 2 \gamma \\
-i \sin 2 \gamma & i \cos 2 \gamma
\end{array}\right)
$$

where under backpropagation the angle $\gamma$ becomes $-\gamma$, therefore $\sin 2 \gamma \rightarrow-\sin 2 \gamma$ as established by Eq. (26). On the other hand, it is easy to check that autocompensating is achieved by a matrix $\sigma_{x}$, which can be implemented by a HWP rotated $\pi / 4$ degrees together and a global phase $e^{i \pi / 2}$, that is,

$$
M^{\prime}=e^{i \pi / 2} H P W_{\pi / 4}=\left(\begin{array}{ll}
0 & 1 \\
1 & 0
\end{array}\right)
$$

therefore, by taking into account the last birrefringent perturbation $q$ we have

$$
\left(\begin{array}{cc}
A_{q} & -i B_{q} \\
-i B_{q} & A_{q}^{*}
\end{array}\right)\left(\begin{array}{ll}
0 & 1 \\
1 & 0
\end{array}\right)\left(\begin{array}{cc}
A_{q} & i B_{q} \\
i B_{q} & A_{q}^{*}
\end{array}\right)=M^{\prime}
$$

and so on, then $S_{1} \ldots S_{q}^{\prime} M^{\prime} S_{q} \ldots S_{1}=M^{\prime}$. This means that the state of the emergent light, back at Charlie's circulators, has been properly restored, albeit a deterministic swapping transformation $M^{\prime}=\sigma_{x}$, which we can account for. In Fig. (5) is shown the implementation of the autocompensating by using a $\mathrm{HPW}_{\pi / 4}$ and an optical circulator to get a closed circuit. The phase shifter $\theta$ to generate quantum states is also shown. In

a)
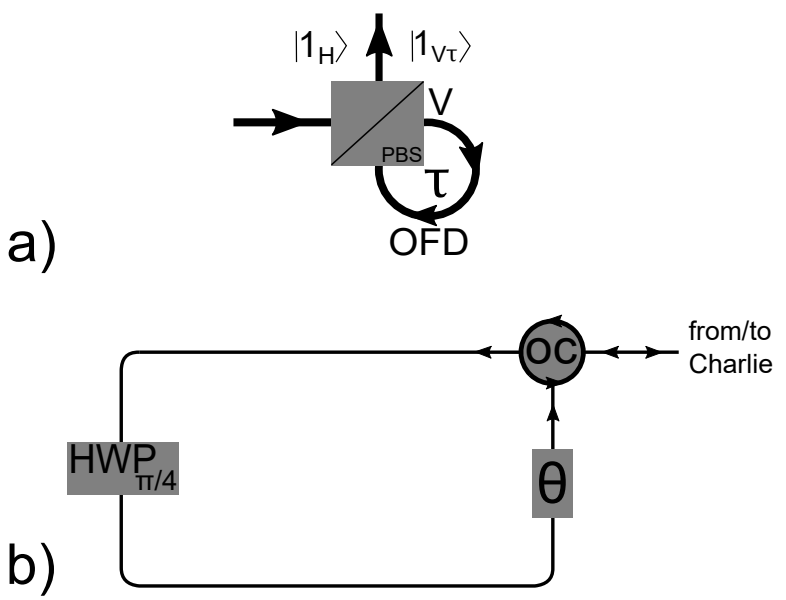

Figure 5 Autocompensating devices and phase modulator for polarization encoding. Photons coming from Charlie enter the local loops at Alice's and Bob's sites. There, a HWP rotated $45^{\circ}$ degrees implement the appropriate autocompensating transformation. Next relative phases are introduced for state generation. 
short, if we take a input polarization state $|L\rangle$, after it has traveled back and forth between Charlie and Alice (Charlie and Bob), it emerges as $\sigma_{x}|L\rangle$, that is, unaltered. The unpredictable changes it would have experienced if it were not for the autocompensating device have been removed. We must note that the usual rectilinear (Z) basis for MDI protocol could not be used for autocompensating purposes because we would have to rotate the state in order to generate different bits, for instance $\left|1_{H}\right\rangle \rightarrow\left|1_{V}\right\rangle$, however, rotations are not autocompensated.

\section{A-MDI-QKD system with codirectional modes}

Space division multiplexing can be also implemented by using multicore fibers (MCFs), that is, by using codirectional modes, therefore, we must also consider these optical fibers for QKD. We must stress that there are many configurations of MCFs [28], that is, different groupings of the cores as for example at the vertices of triangle, a square, or even grouping in pairs [29]. These groupings are enough separated to consider that non modal coupling is produced, however, groups of cores can undergo modal coupling and then are processed by MIMO (Multiple-input Multiple-output) technique. The goal of the groups of cores is to enlarge the transmission capacity by increasing the density of cores. In this work we consider grouping in pairs of singlemode cores, and propose a method for autocompensating the modal coupling between the corresponding modes of two cores [29]. Moreover, an integrated device to measure the Bell states is also proposed. We must stress that sometimes the mentioned cores also are far enough apart and therefore only relative phases are compensated, however, the proposed method can also compensate some possible residual coupling, in fact, mechanical and thermal perturbations can induce mode coupling. The matrix describing an arbitrary perturbation $k(=1, \ldots, q)$ is also given by the matrix $S_{k}$ shown in Eq. (17) where now the modes $e_{1}$ and $e_{2}$ correspond to the fundamental modes of the single-mode cores. Finally, we must stress that polarization is ignored because it is assumed that due to the proximity between cores the polarization changes are common to both cores, that is, a single photon state can be represented as follows

$$
|L\rangle=c_{1}\left|1_{1}\right\rangle+c_{2}\left|1_{2}\right\rangle, \quad\left|1_{j}\right\rangle=c_{H}\left|1_{j H}\right\rangle+c_{V}\left|1_{j V}\right\rangle
$$

with $j=1,2$. This indicates that the polarization state is the same for both cores. From a classical point of view it means that the total optical field can be factorized as $\boldsymbol{e}=\left(c_{1} e_{1}+c_{2} e_{2}\right) \boldsymbol{u}$, with $\boldsymbol{u}=\left(c_{H} \boldsymbol{u}_{H}+c_{V} \boldsymbol{u}_{V}\right)$ an unpredectible polarization unitary vector but non relevant to spatial coupling. In short, the autocompensating method consists of implementing once more the matrix $M=i \sigma_{y}$ used in collinear modes. However, we must stress that unlike of a single-photon QKD we have two photons coming from Alice and Bob, therefore a polarization autocompensating is also required. Such autocompensation will be obtained as indicated in the case of polarization modes, that is, by a HWP rotated $\pi / 4$.

On the other hand, the way to encode information obeys the following spatial scheme: if the photon travels path 1 (core 1 ) that corresponds to bit 0 and if it travels path 2 (core 2 ) that would correspond to bit 1 . The result is that a photon can propagate in a superposition of path 1 and path 2 . Note that this corresponds to the usual dual-rail logic. Formally, $\left|1_{1}\right\rangle \rightarrow$ path 1 and $\left|1_{2}\right\rangle \rightarrow$ path 2 . The experimental implementation parallels that of the previous collinear case, as seen in Fig. 6. There are however a number of changes, especially regarding both the autocompensating process and the Bell-state measurement device (BMD), which in this case is implemented by a optical integrated circuit. Now, each state travels its own path, there being two paths for Alice and two paths for Bob. Each path corresponds by the above encoding to a quantum state. If we label Alice's paths by $a$ and Bob's paths by $b$, we have, for Alice, paths $a_{1}, a_{2}$ and $b_{1}, b_{2}$ for Bob.

In an analogous fashion with respect to the collinear case, Charlie sends a pair of qubits from his ISG, using either a single-photon source or WCPs (decoy states are also required, as usual, for protection against PNS attacks, if WCPs are used). We need to introduce a OFD device in order to introduce a delay between the fundamental states $\left|1_{1}\right\rangle$ and $\left|1_{2}\right\rangle$, for the same reason as before. An example of such a device is shown in Fig. 7. It consists on a pair of photonic lanterns (PL) that extract the modes from the MCF into two parallel SMFs. In one of them, a fiber loop producing a delay $\tau$ is located, as in the MZI. Another photonic lantern takes the photons and puts them into the two cores of the MCF again. Therefore, after the OFDs we have the biphoton state

$$
\left(\frac{\left|1_{1}\right\rangle+\left|1_{2 \tau}\right\rangle}{\sqrt{2}}\right)_{a} \otimes\left(\frac{\left|1_{1}\right\rangle+\left|1_{2 \tau}\right\rangle}{\sqrt{2}}\right)_{b} .
$$

Light travels along two cores of an MCF to meet Alice's and Bob's laboratories. There, local loops contain autocompensating devices and phase modulators. Optical circulators are also required for the local loops of Alice and Bob and to redirect photons to the Bell-state measurement apparatus. Note that circulators do not mix the paths 1 and 2. Although we draw only one circulator, a pair of circulators each one operating in one path is to be understood. 


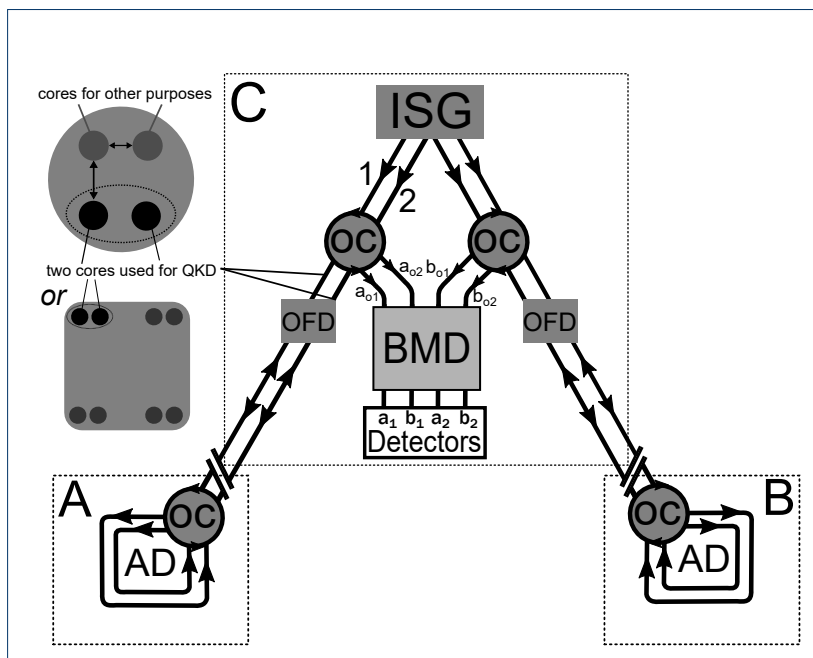

Figure 6 A-MDI-QKD system for codirectional modes. Charlie uses his quantum state generator to send a pair of qubits to each Alice and Bob. After the delays, photons reach the local loops; in them, autocompensating devices and phase modulators are located. Afterwards, light returns to Charlie's six-port OCs, which direct the light towards a optical integrated circuit working as a Bell-state measurement apparatus. We made apparent the fact that we are using codirectional modes by explicitly showing the two paths of the photons, to be understood as two cores of a MCF as shown, although the representation of the system is somewhat schematic.

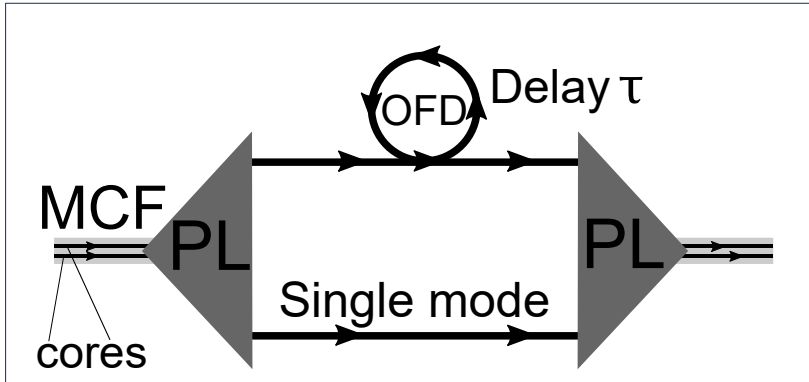

Figure 7 OFD device for codirectional modes. Light comes from the two cores of a MCF and is directed, via, for example, photonic lanterns, to a pair of parallel single-mode fibers. In one of them, an optic fiber delay is placed, introducing a delay $\tau$ in one of the modes. Photons exit the SMFs through another $\mathrm{PL}$ and they are introduced back into the cores of an MCF.

\section{Bell-state measurement device}

Finally, the light that returns to Charlie is directed to a Bell-state measurement device. In this case, such device will be an integrated optical circuit. It is shown in Fig. 8. It is a four-port device; its input fed with the emerging light that comes back to Charlie from Alice's and Bob's sites. Synchronous directional couplers (DC) and phase shifters (PS) implement the required transformations. Finally, at the end of each port, a de- tector is located. The result is a integrated device that is totally analogous to an Innsbruck scheme but on a spatial encoding. Recall that a general synchronous directional coupler implements the following transformation

$$
D(\alpha)=\left(\begin{array}{cc}
\cos \alpha & i \sin \alpha \\
i \sin \alpha & \cos \alpha
\end{array}\right) .
$$

The value of $\alpha=\kappa L$ can be tailored to our choice according to the value of the coupling coefficient $\kappa$ and the coupling length $L$. In this case, the integrated device requires the use of $D(\pi / 4)$ and $D(\pi / 2)$ couplers, along with a pair of $\phi=-\pi / 2$ phase shifters.

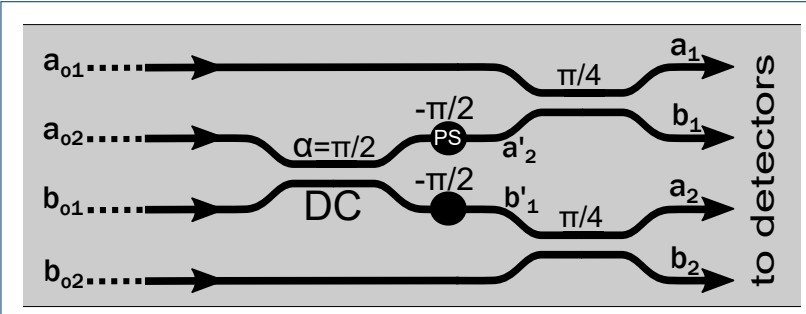

Figure 8 Bell-state measurement device for codirectional modes. A four port optical integrated circuit serves as a Bell-state measurement device for codirectional modes. Photons coming from Charlie's OC enter the device, where three DCs and two phase shifters implement the suitable operations. When light reaches the detectors the Bell states $\left|\Psi^{-}\right\rangle$and $\left|\Phi^{+}\right\rangle$are unambiguously identified, as required by MDI-QKD.

A first $D(\pi / 2)$ coupler is required; it couples inputs $\hat{a}_{o 2}$ and $\hat{b}_{o 1}$. Next, two $D(\pi / 4)$ couplers are needed. They couple, respectively, inputs $a_{o 1}$ and $b_{o 2}$ to intermediate outputs $a_{2}^{\prime}$ and $b_{1}^{\prime}$, respectively. In terms of the photonic absorption operators, the implemented transformations are

$$
\begin{aligned}
& a_{a^{\prime} 2}=\hat{a}_{a o 1} ; \quad a_{a^{\prime} 1}=\hat{a}_{a o 2}, \\
& a_{a o 1}^{\dagger}=\frac{\hat{a}_{a 1}^{\dagger}+i \hat{a}_{b 1}^{\dagger}}{\sqrt{2}} ; \quad \hat{a}_{a^{\prime} 2}^{\dagger}=\frac{i \hat{a}_{a 1}^{\dagger}+\hat{a}_{b 1}^{\dagger}}{\sqrt{2}}, \\
& \hat{a}_{b^{\prime} 1}^{\dagger}=\frac{\hat{a}_{a 2}^{\dagger}+i \hat{a}_{b 2}^{\dagger}}{\sqrt{2}} ; \quad \hat{a}_{b o 2}^{\dagger}=\frac{i \hat{a}_{a 2}^{\dagger}+\hat{a}_{b 2}^{\dagger}}{\sqrt{2}} .
\end{aligned}
$$

As in the previous cases, we will need suitable bases for autocompensation purposes. Therefore, we shall use the following two MUBs

$$
\begin{gathered}
\mathcal{B}_{D}:\left\{\left|1_{D}\right\rangle=\frac{\left|1_{1}\right\rangle+\left|1_{2}\right\rangle}{\sqrt{2}},\left|1_{A}\right\rangle=\frac{\left|1_{1}\right\rangle-\left|1_{2}\right\rangle}{\sqrt{2}}\right\}, \\
\mathcal{B}_{C}:\left\{\left|1_{L}\right\rangle=\frac{\left|1_{1}\right\rangle+i\left|1_{2}\right\rangle}{\sqrt{2}},\left|1_{R}\right\rangle=\frac{\left|1_{1}\right\rangle-i\left|1_{2}\right\rangle}{\sqrt{2}}\right\} .
\end{gathered}
$$


Moreover, the transformation between absorption operators $\hat{a}$ in both bases is given by

$$
\hat{a}_{\left(\begin{array}{l}
D \\
A
\end{array}\right)}=\frac{1}{\sqrt{2}}\left(\hat{a}_{1} \pm \hat{a}_{2}\right) ; \quad \hat{a}_{\left({ }_{R}^{L}\right)}=\frac{1}{\sqrt{2}}\left(\hat{a}_{1} \mp i \hat{a}_{2}\right)
$$

Let us assume, for instance, that Alice and Bob prepare the input state $\left|L_{n f}\right\rangle=\left|1_{a o D} 1_{b o D}\right\rangle=$ $a_{a o D}^{\dagger} a_{v o D}^{\dagger}|00\rangle$, then by taking into account Eq. (36) we obtain the state

$$
\frac{1}{2}\left\{\left|1_{a o 1} 1_{b o 1}\right\rangle+\left|1_{a o 1} 1_{b o 2}\right\rangle+\left|1_{a o 2} 1_{b o 1}\right\rangle+\left|1_{a o 2} 1_{b o 2}\right\rangle\right\}
$$

With this in hand, together with the transformations in Eq. (33), we can make a Bell-state analysis of the device in Fig. (8). Thus, after a long but straightforward calculation, we will find that the output state is a superposition of states which do not produce coincidences and states producing coincidences, that is, $|L\rangle=\left|L_{n c}\right\rangle+\left|L_{c}\right\rangle$, with

$$
\begin{aligned}
& \left|L_{n c}\right\rangle=i \frac{\sqrt{2}}{4}\left(\left|2_{a 1}\right\rangle+\left|2_{b 1}\right\rangle+\left|2_{a 2}\right\rangle+\left|2_{b 1}\right\rangle\right) \\
& \left|L_{c}\right\rangle=\frac{i}{2}\left(\left|1_{a 1} 1_{a 2}\right\rangle+\left|1_{b 1} 1_{b 2}\right\rangle\right) \equiv i \frac{\sqrt{2}}{2}\left|\Phi^{+}\right\rangle
\end{aligned}
$$

Now, let us assume that Alice and Bob send back to Charlie orthogonal states but in the same (diagonal basis), i.e the input state is

$$
\left|L_{f}\right\rangle=a_{a o D}^{\dagger} a_{b o A}^{\dagger}|00\rangle .
$$

In this case, by identical procedure, we will obtain number states $|2\rangle$ and the following Bell state $\left|\Psi^{-}\right\rangle$, with probability $1 / 2$ :

$$
\left|\Psi^{-}\right\rangle=\frac{1}{\sqrt{2}}\left(\left|1_{b 1} 1_{a 2}\right\rangle-\left|1_{a 1} 1_{b 2}\right\rangle\right)
$$

Thus, the optical integrated circuit we propose works as a Bell-state analyser, as it should, and therefore the MDI protocol can be implemented. Next, we present the autocompensating method.

\section{Autocompensating method}

As in the collinear case, unpredictable coupling between modes of adjacent cores of an MCF results in a series of perturbations $S_{k}$, each which can be characterized again as a $\mathrm{SU}(2)$ matrix with the same form as Eq. (17). It represents, again, the perturbation associated to a short length, in the $z$-direction, of the core the photons travel along. The total matrix is again given by $S=S_{q} S_{q-1} \ldots S_{2} S_{1}$. Importantly, these matrices have the same symmetries as the ones of collinear modes. Consequently, we need to find a way to implement the transformation $M=i \sigma_{y}$ on codirectional modes, so that we obtain $S_{k} M S_{k}=M$ for each perturbation $k$. There are a number of ways to implement such transformation. Here, we choose a simple one, consisting of a directional coupler together with phase shifter introducing a phase $\pi$, placed in a closed circuit, as shown in Fig. (9). Specifically, for the DC,

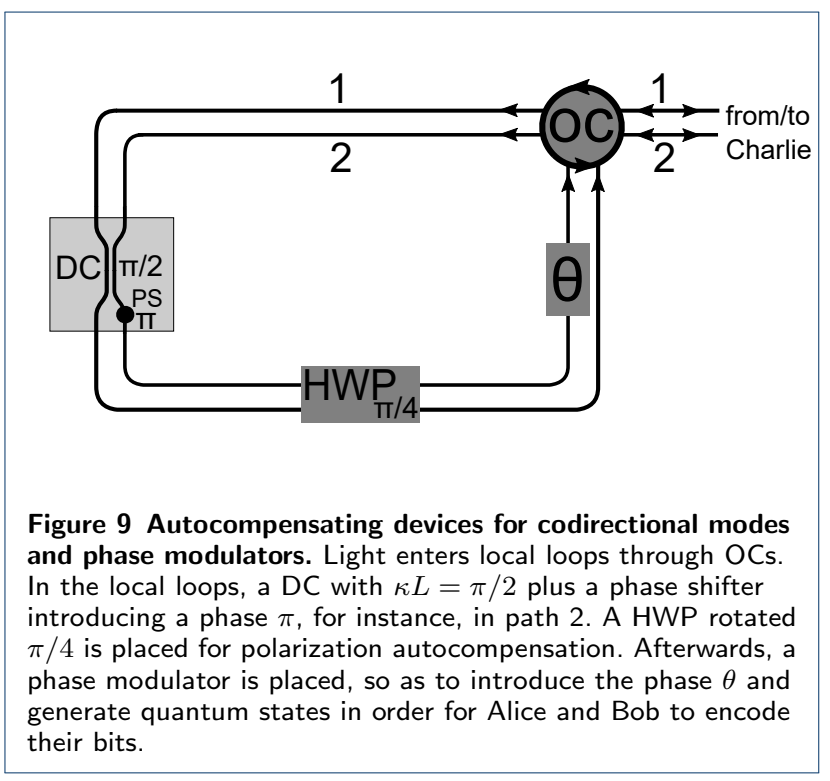

we set $\alpha=\pi / 2$, so from Eq. (32) we obtain

$$
D(\kappa)=i\left(\begin{array}{ll}
0 & 1 \\
1 & 0
\end{array}\right)
$$

Next, place a $\pi$ phase shifter on the path 2 and thus we obtain the transformation

$$
U(\pi) D(\pi / 2)=i\left(\begin{array}{cc}
0 & 1 \\
-1 & 0
\end{array}\right)
$$

which is just what we wanted, up to a harmless global phase $\pi / 2$. Note that the DC implements a swapping operation. This means that it exchanges the physical modes (not their identity), in the sense that the retarded mode will now follow the path which has no delay, and the advanced mode will be delayed. Thus, modes reach Charlie's Bell-state measurement apparatus at the same time and autocompensation is achieved. This is analogous to what happened in the collinear case.

Finally, a HWP is also included for autocompensate polarization because the perturbations in the optical fiber linking points Alice-Charlie and the optical fiber 
linking point Bob-Charlie will be different. Obviously, we can take advantage of this autocompensating polarization to use two different QKD channels, that is, collinear modes in $\mathrm{H}$ and $\mathrm{V}$ polarizations (channels), and therefore doubling the bit rate. We only need to separate the polarization channels (by using, for instance, PBSs) and to place two BMDs in the A-MDIQKD system.

\section{Conclusions}

We have shown how to implement a A-MDI-QKD protocol in different settings according to the kind of optical fiber used for space division multiplexing, that is, few-mode optical fibers maintaining polarization modes (PM-FMF) or maintaining space modes (SMFMF) and MCFs. In particular, we have proposed three systems for A-MDI-QKD by using collinear, polarizations and codirectional modes. Discrete and/or integrated components have been used for both measuring Bell states and achieving autocompensation. As such fibers are assumed to be the links of present and near-future optical networks, then implementation of QKD in them and in a plug-and-play fashion would offer clear practical benefits, as for example, to use several channels for MDI-QKD what would in turn compensate the losses and therefore increase the bit rate.

\section{Abbreviations}

QKD: Quantum Key Distribution; MDI-QKD:

Measurement-Device-Independent Quantum Key Distribution; MCF: Multicore Optical Fiber; FMF: Few-Mode Fiber; A-MDI-QKD:

Autocompensating Measurement-Device-Independent Quantum Key Distribution; HG: Hermite-Gauss; PM-FMF: Polarization-Maintaining Few Mode Fibers; SP-SMF: Space-Maintaining Single Mode Fiber; SPDC: Spontaneous Parametric Down-Conversion; WCP: Weak Coherent Pulses; PNS: Photon Number Splitting; ISG: Initial States Generation; OC: Optical Circulators; OFD: Optical Fiber Delay; MZI: Mach-Zehnder Interferometer; DP: Dove Prism; BS: Beam Splitter; PBS: Polarizing Beam Splitter; CLC: Cylindrical Lens Converter; DC: Directional Coupler; HWP: Half-Wave Plate; PS: Phase Shifter; BMD: Bell-state Measurement Device; MIMO: Multiple-input Multiple-output.

Availability of data and materials

Not applicable.

\section{Competing interests}

The authors declare that they have no competing interests.

\section{Funding}

Financial support by Xunta de Galicia, Consellería de Educación, Universidades e FP, through a grant Consolidation-GRC

Ref.-ED431C2018/11, and two predoctoral grants (D. Balado, 2017 and G. Carral, 2020), both co-financed by the European Social Fund.

\section{Author's contributions}

$\mathrm{JL}$ and XP-B conceived the presented idea. All authors developed the methodology, discussed the results, wrote the paper, and read and approved the final manuscript.

\section{Author details}

Quantum Materials and Photonics Research Group, Optics Area, Applied Physics Department, Faculty of Physics / Faculty of Optics and Optometry, University of Santiago de Compostela, E-15782 Santiago de Compostela, Galicia, Spain

\section{Acknowledgements}

Not applicable

\section{References}

1. Bai, N., Ip, E., Huang, Y.-K., Mateo, E., Yaman, F., Li, M.-J. Bickham, S., Ten, S., Liñares, J., , Montero, C., Moreno, V., Prieto, X., Tse, V., Chung, K.M., Lau, A.P.T., Tam, H.-Y., Lu, C., Luo, Y., Peng, G.-D., Li, G., Wang, T.: Mode-division multiplexed transmission with inline few-mode fiber amplifier. Opt. Express 20(3), 2668-2680 (2012)

2. Saitoh, K., Matsuo, S.: Multicore fiber technology. J. Lightwave Tech. 34, 55-66 (2016)

3. Gisin, N., Ribordy, G., Tittel, W., Zbinden, H.: Quantum cryptography. Rev. Mod. Phys. 74, 145-195 (2002)

4. Pirandola, S., Andersen, U.L., Banchi, L., Berta, M., Bunandar, D., Colbeck, R., Englund, D., Gehring, T., Lupo, C., Ottaviani, C., Pereira, J., Razavi, M., Shaari, J.S., Tomamichel, M., Usenko, V.C., Vallone, G., Villoresi, P., Wallden, P.: Advances in Quantum Cryptography (2019). 1906.01645

5. Zhao, Y., Fung, C.-H.F., Qi, B., Chen, C., Lo, H.-K.: Quantum hacking: Experimental demonstration of time-shift attack against practical quantum-key-distribution systems. Phys. Rev. A 78, 042333 (2008)

6. Lydersen, L., Wiechers, C., Wittmann, C., Elser, D., Skaar, J., Makarov, V.: Hacking commercial quantum cryptography systems by tailored bright illumination. Nature Photonics 4(10), 686-689 (2010)

7. Gerhardt, I., Liu, Q., Lamas-Linares, A., Skaar, J., Kurtsiefer, C., Makarov, V.: Full-field implementation of a perfect eavesdropper on a quantum cryptography system. Nature Communications 2(1) (2011)

8. Lo, H.-K., Curty, M., Qi, B.: Measurement-device-independent quantum key distribution. Phys. Rev. Lett. 108, 130503 (2012)

9. Hwang, W.-Y.: Quantum key distribution with high loss: Toward global secure communication. Phys. Rev. Lett. 91, 057901 (2003)

10. Dellantonio, L., Sørensen, A.S., Bacco, D.: High-dimensional measurement-device-independent quantum key distribution on two-dimensional subspaces. Phys. Rev. A 98, 062301 (2018). doi:10.1103/PhysRevA.98.062301

11. Xu, F., Curty, M., Qi, B., Lo, H.-K.: Practical aspects of measurement-device-independent quantum key distribution. New Journal of Physics 15(11), 113007 (2013)

12. Yan, H., Li, S., Xie, Z., Zheng, X., Zhang, H., Zhou, B.: Design of panda ring-core fiber with 10 polarization-maintaining modes. Photon. Res. 5, 1-5 (2017)

13. Bethune, D.S., Risk, W.P.: Autocompensating quantum cryptography. New Journal of Physics 4, 42-42 (2002)

14. Balado, D., Liñares, J., Prieto-Blanco, X., Barral, D.: Phase and polarization autocompensating $\mathrm{N}$-dimensional quantum cryptography in multicore optical fibers. JOSA B 34, 2793-2803 (2019)

15. Hu, X.-M., Xing, W.-B., Liu, B.-H., He, D.-Y., Cao, H., Guo, Y., Zhang, C., Zhang, H., Huang, Y.-F., Li, C.-F., Guo, G.-C.: Efficient distribution of high-dimensional entanglement through $11 \mathrm{~km}$ fiber. Optica 7, 738-743 (2020)

16. Ip, E., Milione, G., Li, M.-J., Cvijetic, N., Kanonakis, K., Stone, J., Peng, G., Prieto, X., Montero, C., Moreno, V., , Liñares, J.: Sdm transmission of real-time $10 \mathrm{gbe}$ traffic using commercial sfp + transceivers over $0.5 \mathrm{~km}$ elliptical-core few-mode fiber. Opt. Express 23 , 17120-17126 (2015)

17. Muller, A., Herzog, T., Huttner, B., Tittel, W., Zbinden, H., Gisin, N.: "plug and play" systems for quantum cryptography. Applied Physics Letters 70(7), 793-795 (1997)

18. Sibson, P., Kennard, J.E., Stanisic, S., Erven, C., O'Brien, J.L., Thompson, M.G.: Integrated silicon photonics for high-speed quantum key distribution. Optica 4(2), 172-177 (2017)

19. Dynes, J.F., Kindness, S.J., Tam, S.W.-B., Plews, A., Sharpe, A.W. Lucamarini, M., Fröhlich, B., Yuan, Z.L., Penty, R.V., Shields, A.J.: Quantum key distribution over multicore fiber. Opt. Express 24(8), 8081-8087 (2016)

20. Cañas, G., Vera, N., Cariñe, J., González, P., Cardenas, J., Connolly, P.W.R., Przysiezna, A., Gómez, E.S., Figueroa, M., Vallone, G., Villoresi, P., da Silva, T.F., Xavier, G.B., Lima, G.: High-dimensional decoy-state quantum key distribution over multicore telecommunication fibers. Phys. Rev. A 96, 022317 (2017) 
21. Riesen, N., Gross, S., Love, J.D., Sasaki, Y., Withford, M.J.: Monolithic mode-selective few-mode multicore fiber multiplexers. Scientific Rep. 7, 69711 (2017)

22. Labroille, G., Barré, N., Pinel, O., Denolle, B., Lenglé, K., Garcia, L., Jaffrès, L., Jian, P., Morizur, J.-F.: Characterization and applications of spatial mode multiplexers based on multi-plane light conversion. Opt. Fiber Technol. 35, 93-99 (2017)

23. Liñares, J., Nistal, M.C., Barral, D., Moreno, V.: Optical field-strength polarization of two-mode single-photon states. European Journal of Physics 31(5), 991-1005 (2010)

24. Beijersbergen, M.W., Allen, L., van der Veen, H.E.L.O., Woerdman, J.P.: Astigmatic laser mode converters and transfer of orbital angular momentum. Opt. Commun. 96, 123-132 (1993)

25. Hu, M., Zhang, L., Guo, B., Li, J.: Polarization-based plug-and-play measurement-device-independent quantum key distribution. Optical and Quantum Electronics 51(1), 22 (2019)

26. Ip, E., Milione, G., Li, M.-J., Cvijetic, N., Kanonakis, K., Stone, J., Peng, G., Prieto, X., Montero, C., Moreno, V., Liñares, J.: Sdm transmission of real-time $10 \mathrm{gbe}$ traffic using commercial sfp + transceivers over $0.5 \mathrm{~km}$ elliptical-core few-mode fiber. Opt. Express 23 . 240421 (2015)

27. Balado-Souto, D., Liñares, J., Prieto-Blanco, X.: Phase auto-compensating high-dimensional quantum cryptography in elliptical-core few-mode fibres. J.Mod.Opt. 66, 947-957 (2019)

28. Hayashi, T., Nakanishi, T.: Multi-core optical fibers for the next-generation communications. SEI Technical Review 86, 23-28 (2018)

29. Sakamoto, T., Mori, T., Wada, M., Yamamoto, T., Yamamoto, F., Nakajima, K.: Strongly-coupled multi-core fiber and its optical characteristics for mimo transmission systems. Opt. Fiber Technol. 35 8-18 (2016) 


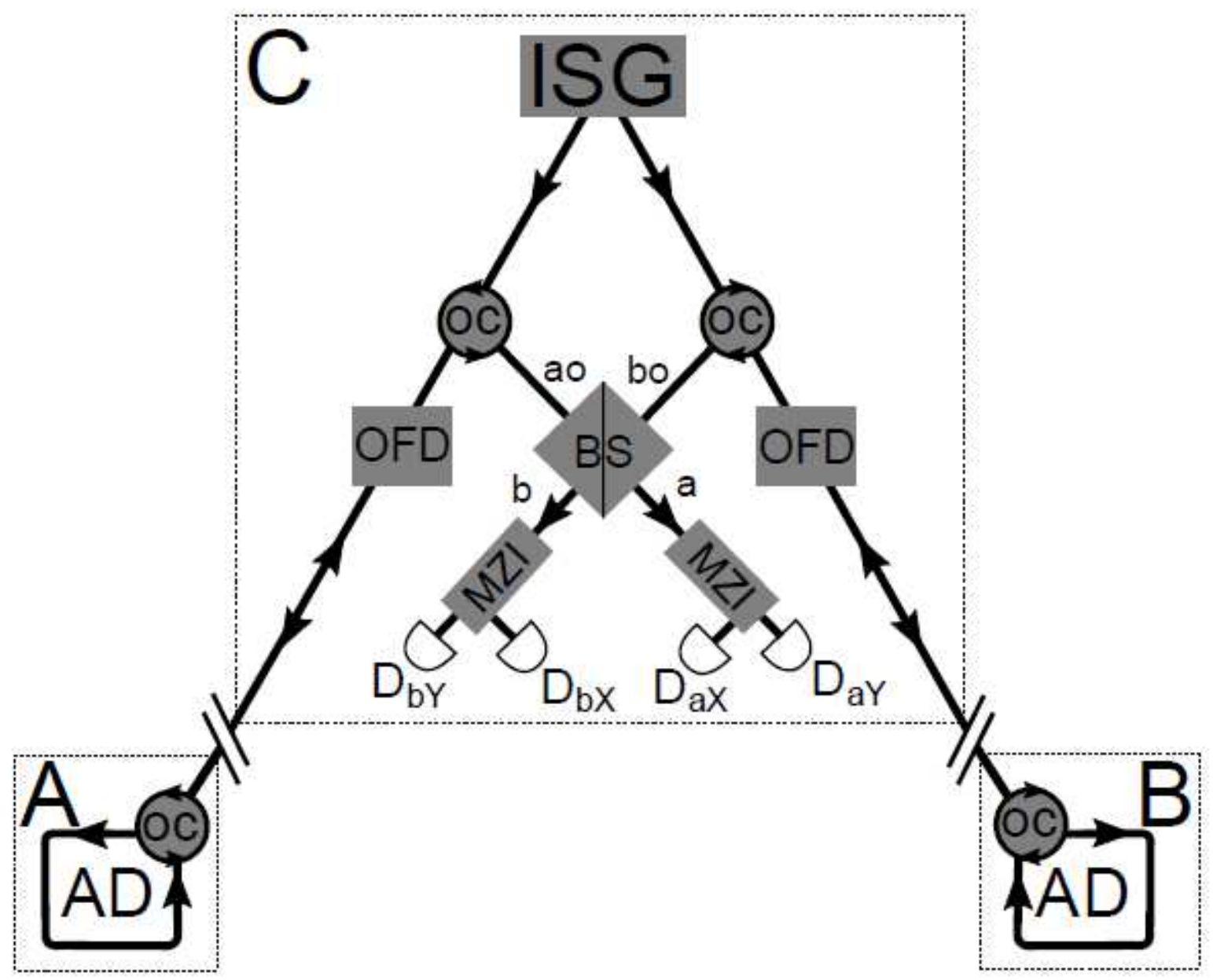

Figure 1

A-MDI-QKD system for collinear modes. Light comes from Charlie along PM-FMF optical bers. States are delayed by OFDs. They arrive at Alice's and Bob's laboratories, where they travel along local loops equipped with autocompensating devices (AD) and phase modulators. Local loop structure is symbolized by a loop with $A D$ inside. Light returns to Charlie, and optical circulators $(O C)$ direct the photons towards a Bell-state measurement apparatus composed by a BS and two MZIs working as mode sorters, together with the corresponding detectors. 


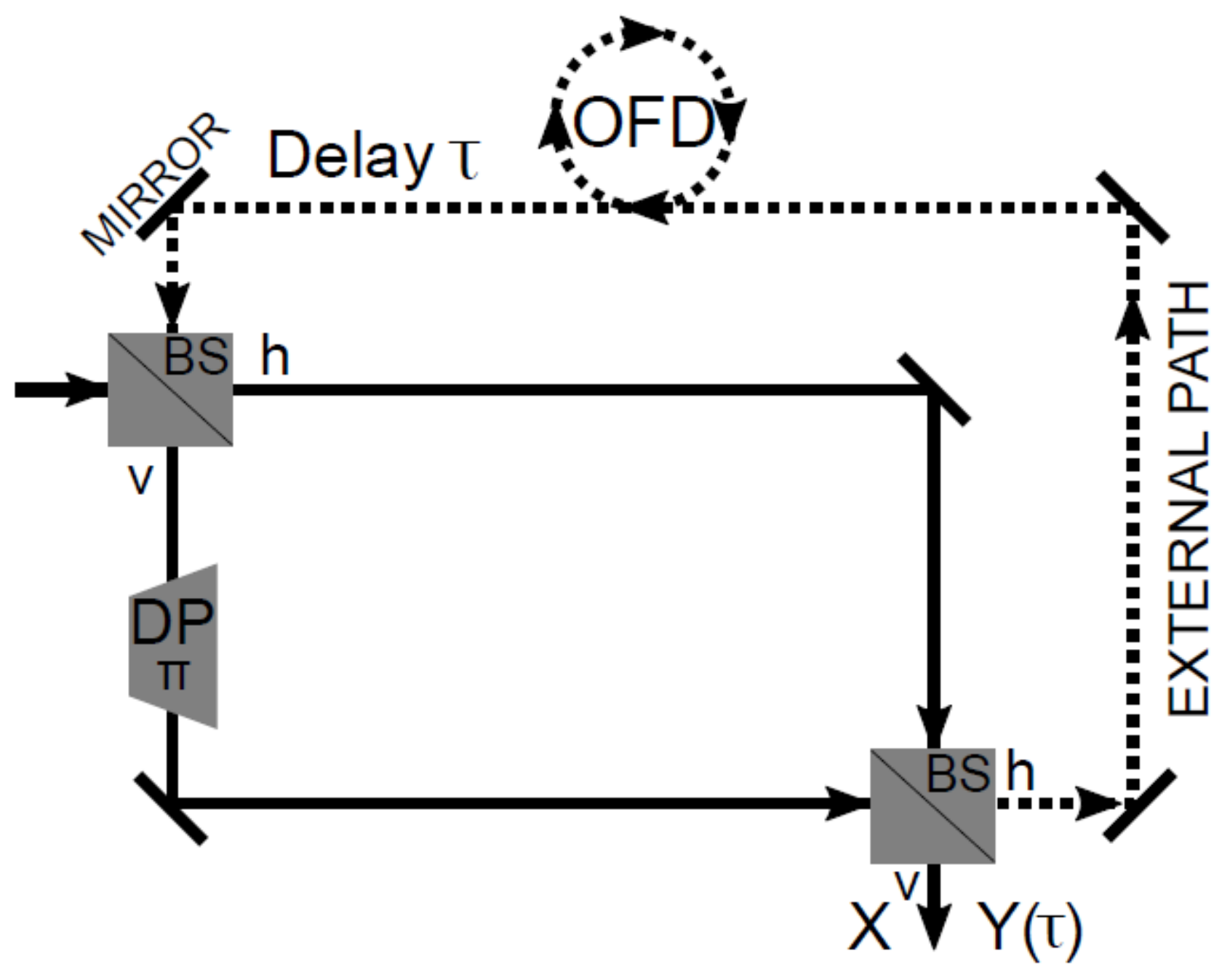

Figure 2

Scheme of a MZI operating with HG modes. A Mach-Zehnder Interferometer together with a Dove prism in one of its arms operates as a HG mode sorter. At the output of the last BS, the X mode emerges on the $v$ path and the $\mathrm{Y}$ mode on the $\mathrm{h}$ path. This modes can be either redirected to detectors, as in Figure 1, to project onto Bell states, or the $\mathrm{Y}$ mode can be redirected into a external path (dashed line), introducing a delay $\tau$ between modes, that is, $\mid 1 X>$ and $\mid 1 Y \tau>$. Thus, such MZls can be used as 'polarizing' beam splitters (thinking of $X$ and $Y$ as polarizations) for Bell-state detection as well as OFD devices. 


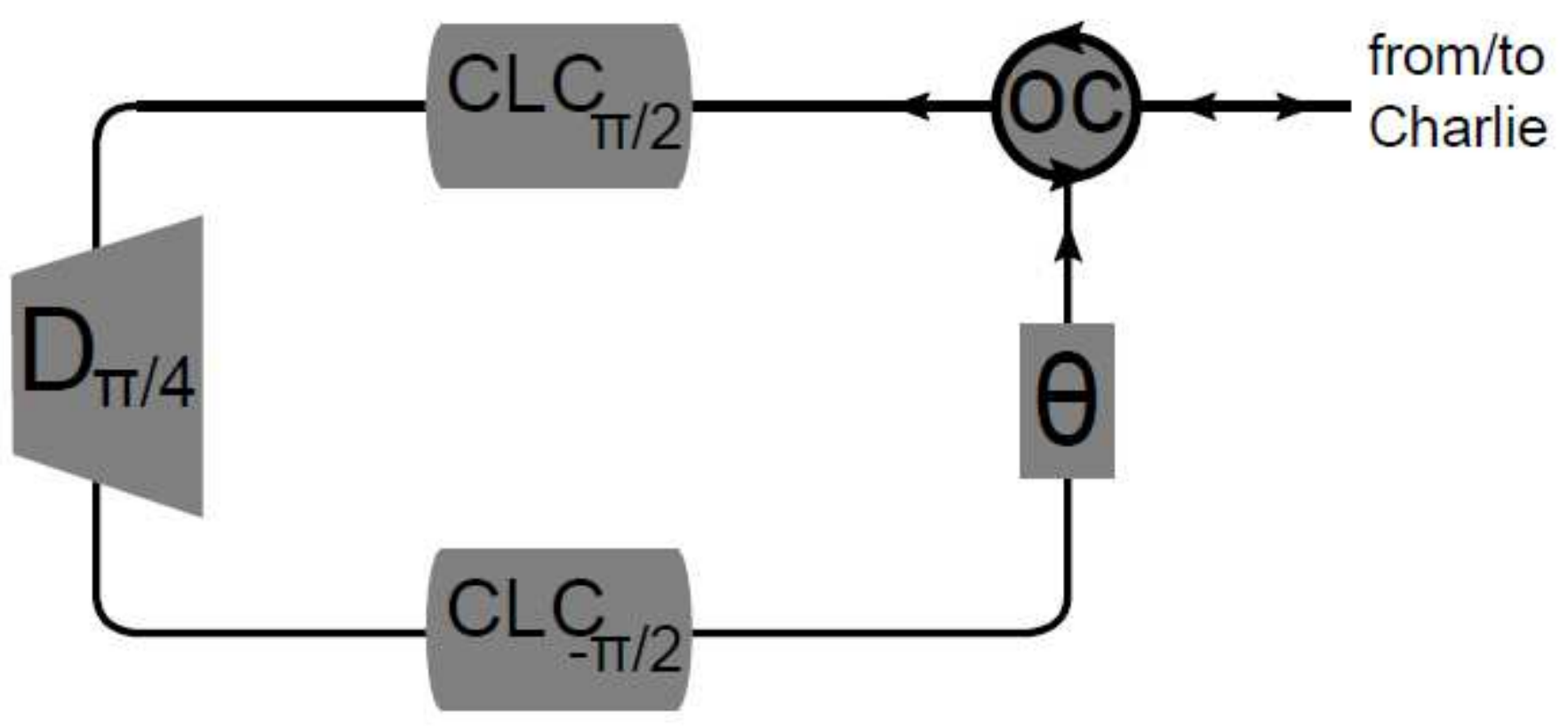

Figure 3

Autocompensating devices for collinear modes and state generation by phase modulation. At Alice's and Bob's sites local loops equipped with autocompensating devices and phase modulators are located. The combination of two CLCs and a Dove prism, each one with a determinate orientation, implements the matrix - $\sigma y$, which suffices to autocompensate for perturbations due to modal coupling. The phase modulator introduces a phase $\theta$ by which to send photons back to Charlie in one of the two MUBs B1 and B2 described above. 


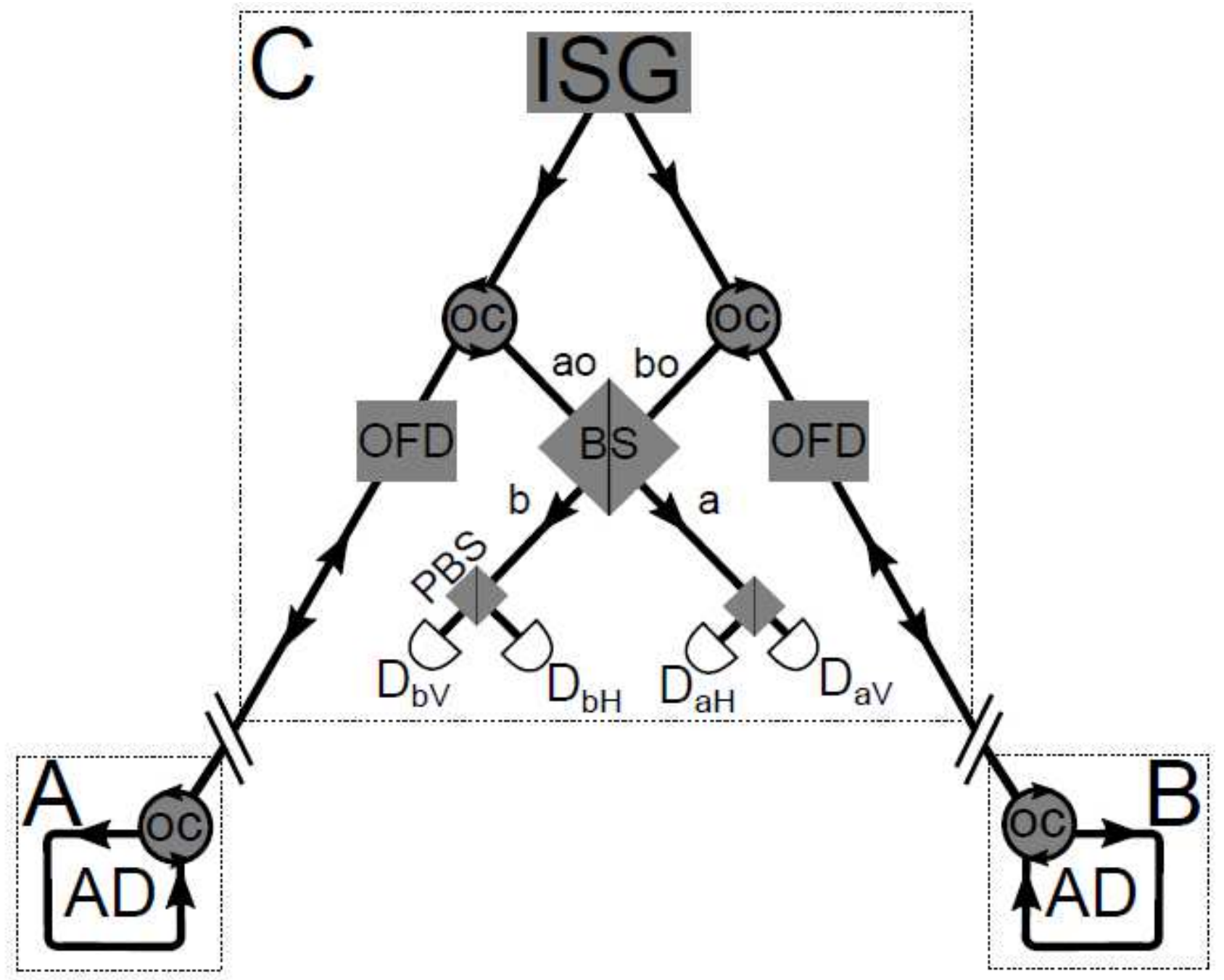

Figure 4

A-MDI-QKD system for polarization encoding. Charlies generates suitable polarization states and launches them towards Alice and Bob. They autocompensate and modulate the relative phase between the horizontal and vertical components so as to encode their information. Light comes back to Charlie. Circulators redirect it to a polarization Bell-state analyser (Innsbruck scheme) 


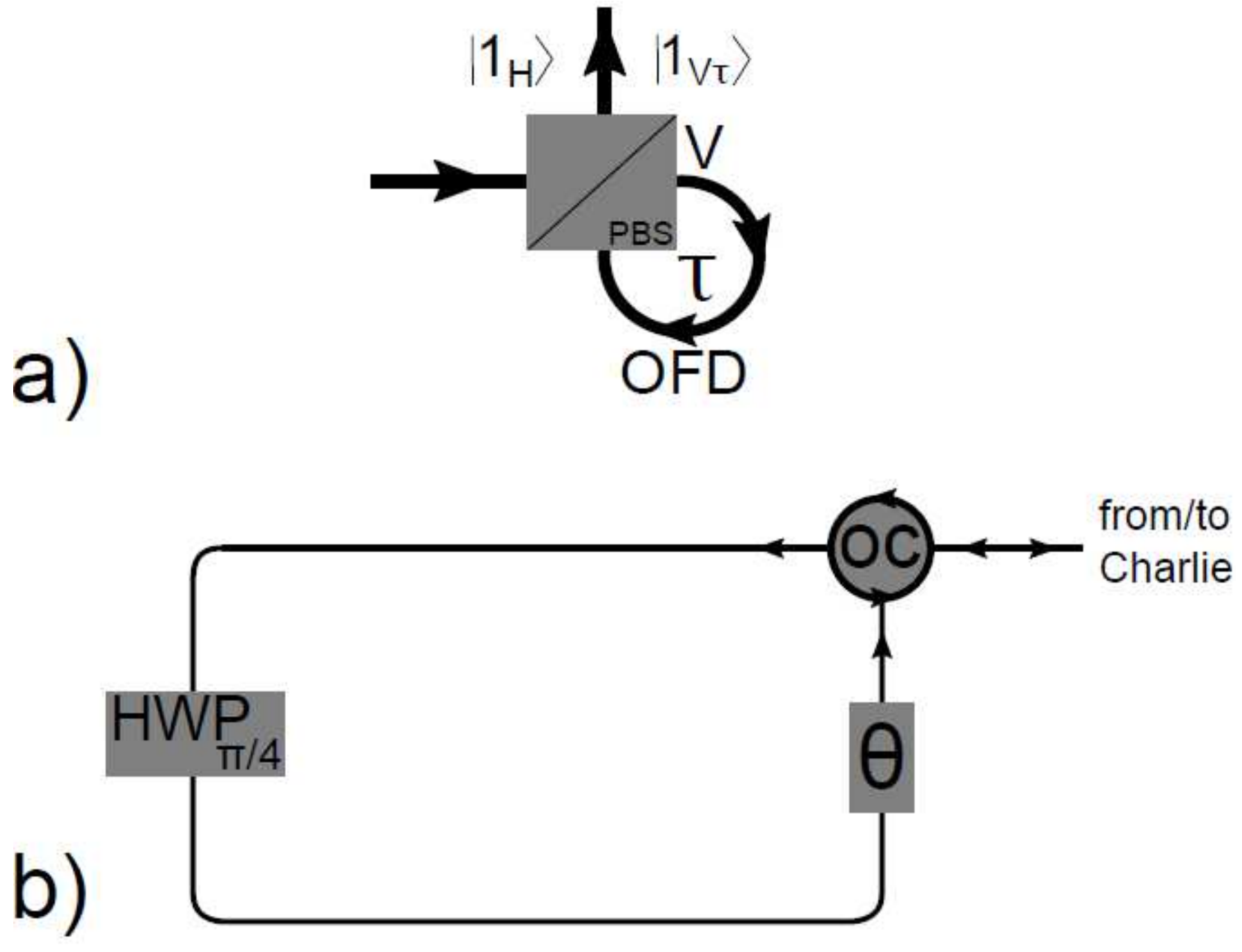

Figure 5

Autocompensating devices and phase modulator for polarization encoding. Photons coming from Charlie enter the local loops at Alice's and Bob's sites. There, a HWP rotated 450 degrees implement the appropriate autocompensating transformation. Next relative phases are introduced for state generation. 


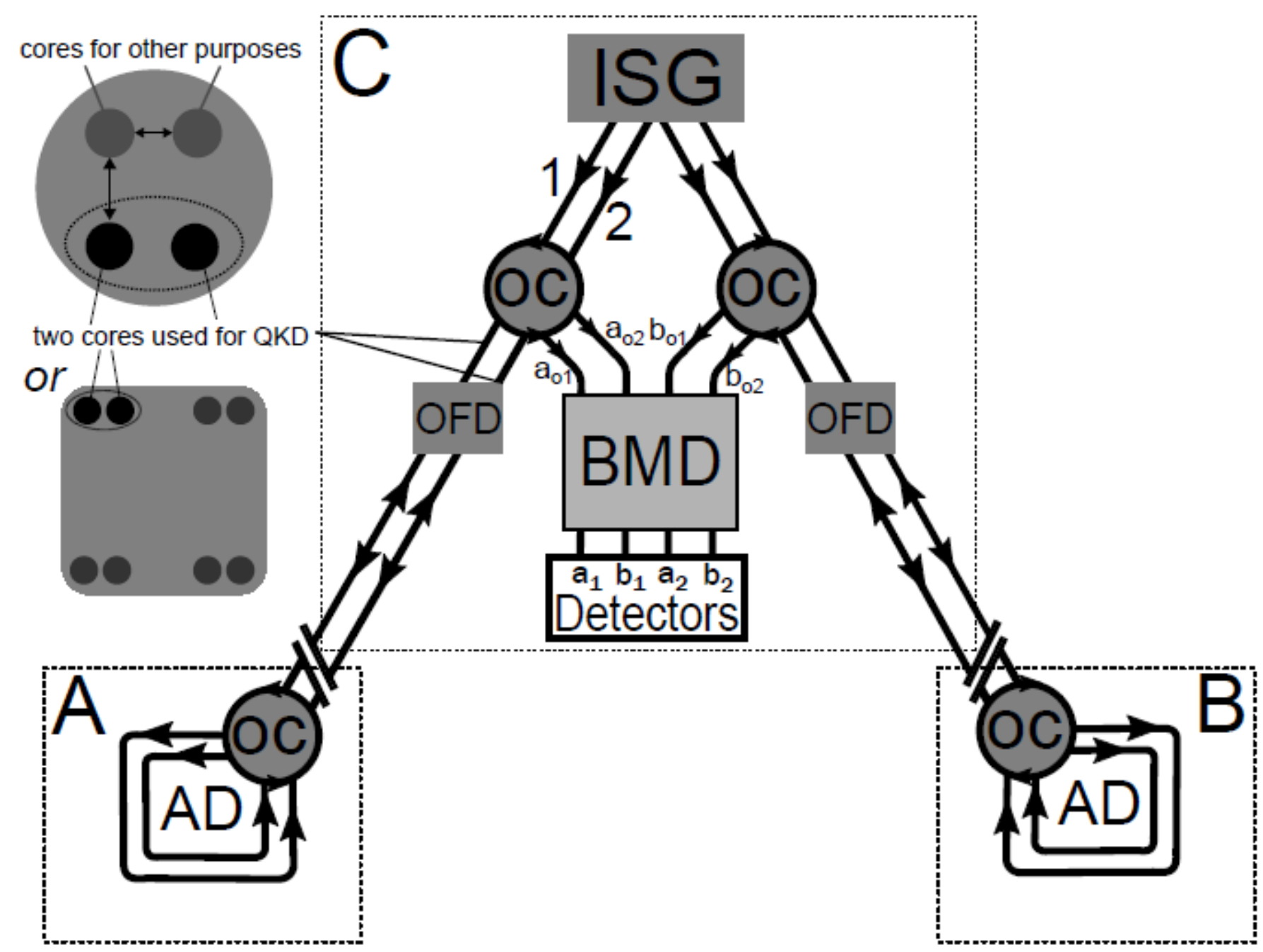

Figure 6

A-MDI-QKD system for codirectional modes. Charlie uses his quantum state generator to send a pair of qubits to each Alice and Bob. After the delays, photons reach the local loops; in them, autocompensating devices and phase modulators are located. Afterwards, light returns to Charlie's six-port OCs, which direct the light towards a optical integrated circuit working as a Bell-state measurement apparatus. We made apparent the fact that we are using codirectional modes by explicitly showing the two paths of the photons, to be understood as two cores of a MCF as shown, although the representation of the system is somewhat schematic. 


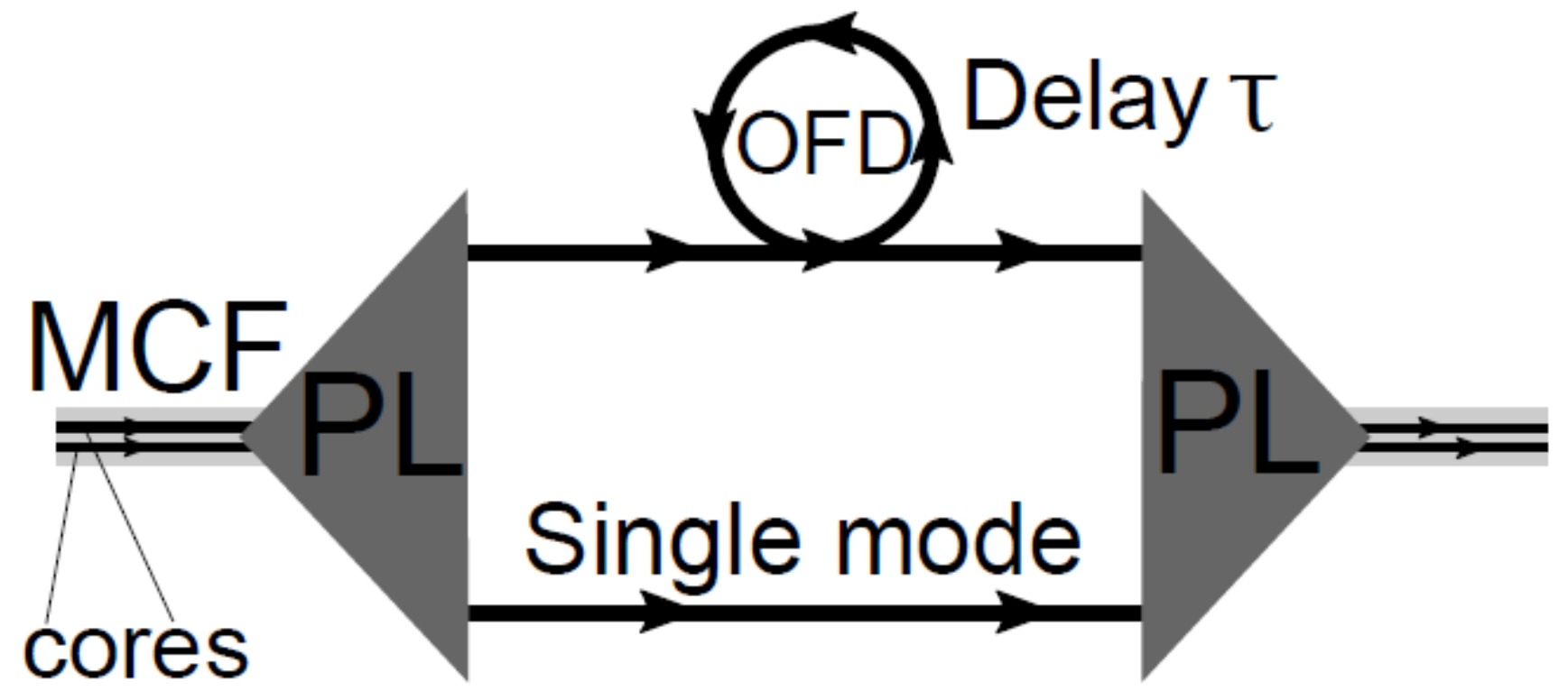

Figure 7

OFD device for codirectional modes. Light comes from the two cores of a MCF and is directed, via, for example, photonic lanterns, to a pair of parallel single-mode fibers. In one of them, an optic fiber delay is placed, introducing a delay $\tau$ in one of the modes. Photons exit the SMFs through another PL and they are introduced back into the cores of an MCF.

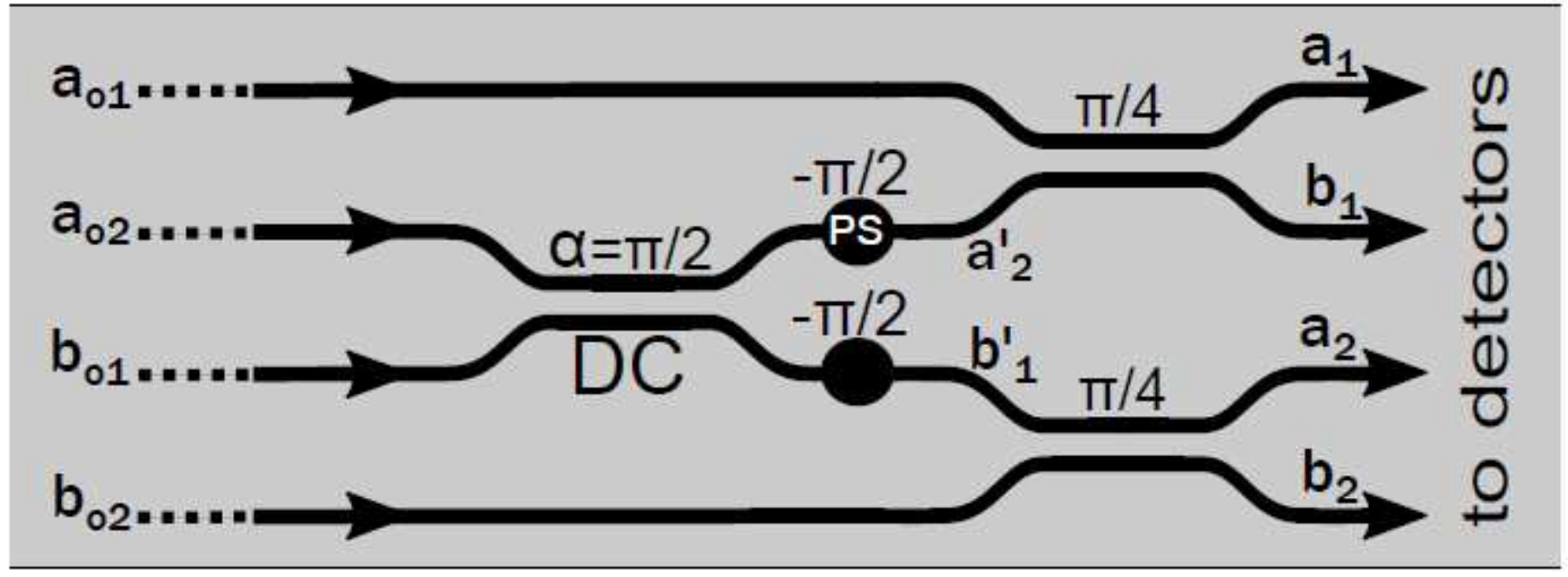

Figure 8

Bell-state measurement device for codirectional modes. A four port optical integrated circuit serves as a Bell-state measurement device for codirectional modes. Photons coming from Charlie's OC enter the device, where three DCs and two phase shifters implement the suitable operations. When light reaches the detectors the Bell states $\mid \Psi$ - > and $\mid \Phi+>$ are unambiguously identified, as required by MDI-QKD. 


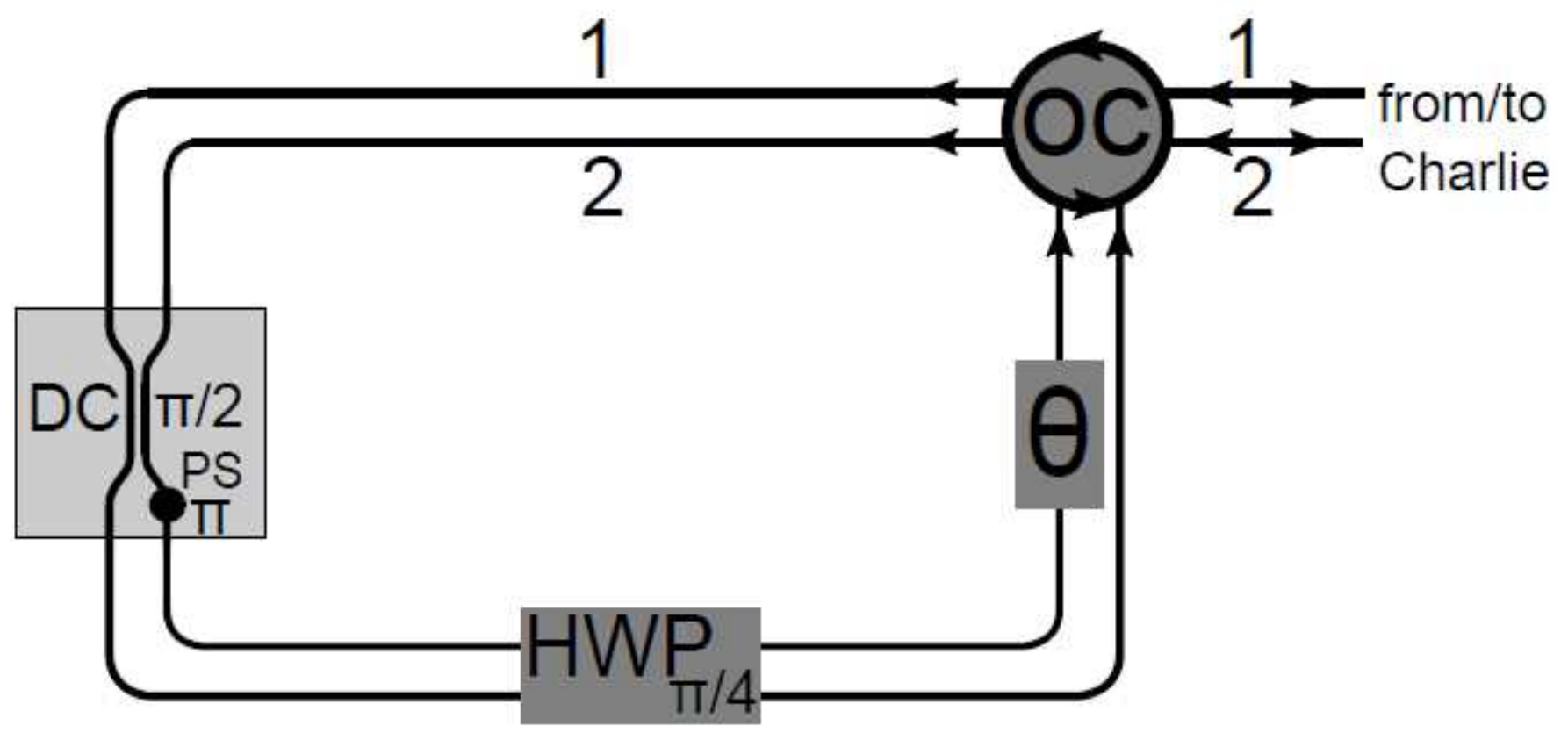

Figure 9

Autocompensating devices for codirectional modes and phase modulators. Light enters local loops through OCs. In the local loops, a DC with $\mathrm{kL}=\pi / 2$ plus a phase shifter introducing a phase $\pi$, for instance, in path 2. A HWP rotated $\pi / 4$ is placed for polarization autocompensation. Afterwards, a phase modulator is placed, so as to introduce the phase $\theta$ and generate quantum states in order for Alice and Bob to encode their bits. 